\title{
SOME SELF-ADJOINT QUANTUM SEMIMARTINGALES
}

\author{
ALEXANDER C. R. BELTON
}

\section{Introduction}

The following quotation [21, Introduction], with which we agree strongly, refers to the Hudson-Parthasarathy theory of quantum semimartingales [10].

Examples of symmetric quantum semimartingales are easy to find, but essentially self-adjoint quantum semimartingales have, in general, proved elusive. This is not unexpected since a quantum semimartingale is in some sense an indefinite integral of a family of unbounded operators, and strong conditions are required to ensure that the sum of even two unbounded self-adjoint operators is self-adjoint.

The vacuum-adapted theory makes a striking contrast to this: the gauge integral preserves self-adjointness. More precisely, if $H$ is a vacuum-adapted, self-adjoint process then

$$
U_{s}:=\mathbb{R}_{+} \ni t \mapsto I+\int_{0}^{t}\left(e^{i s H(r)}-I\right) d A_{r}^{\circ}
$$

is a unitary process for all $s \in \mathbb{R}$ and $\left(U_{s}(t): s \in \mathbb{R}\right)$ is a strongly continuous, one-parameter unitary group for all $t \geqslant 0$; unitarity is a simple consequence of the multiplicativity of the vacuum-adapted gauge integral. (It seems necessary to impose conditions on $H$ in order to ensure the integrand in (1.1) is measurable and vacuum-adapted.) Furthermore, in the strong sense,

$$
\lim _{s \rightarrow 0} \frac{U_{s}(t)-I}{i s}=\int_{0}^{t} H(r) d A_{r}^{\circ} \text { for all } t \geqslant 0,
$$

where the integral has its maximal domain.

One way of viewing this result is as the commutativity of the maps

$$
H \mapsto e^{i H}-I \quad \text { and } \quad H \mapsto \int H d A^{\circ}
$$

on a large class of vacuum-adapted, self-adjoint processes. This relationship extends to a functional Itô formula: if $f: \mathbb{R} \rightarrow \mathbb{C}$ is a bounded, Borel-measurable function then

$$
f\left(\int H d A^{\circ}\right)=f(0)+\int(f(H)-f(0)) d A^{\circ} .
$$

If $H$ is a self-adjoint process which is adapted in the sense of Hudson and Parthasarathy, the isomorphism [6] between vacuum-adapted semimartingales and those adapted in the HP-sense yields, in many cases, a self-adjoint process $K$ such that

$$
K=\int(H-K) d A^{\circ}
$$

(Some care must be taken interpreting this integral; we follow Attal [3].)

Received 14 December 2004; revised 30 June 2005.

2000 Mathematics Subject Classification 81S25, 47D06, 47A56. 
With either type of adaptedness, the processes obtained may be perturbed by bounded, self-adjoint quantum semimartingales to produce further examples.

Although our central idea is very simple, certain technicalities have to be addressed to ensure that $e^{i s H}$ inherits adaptedness and measurability properties from the self-adjoint process $H$. It appears, for $H$ unbounded, that neither problem has been examined before.

We prove that various conditions for measurability, given in terms of the associated unitary groups, resolvents and spectral measures, are all equivalent and we provide a sufficient condition for these to hold which may readily be verified for various processes of interest in the quantum stochastic framework.

The adaptedness condition we adopt is the strong generalization of that which holds in the bounded case: operators are required to have closure equal to the ampliation by a particular projection, which is the identity in the usual theory and equal to the vacuum projection for the vacuum-adapted case. (As some interest has been shown in other possibilities, and it involves no extra working, we consider these as well.) We show that this requirement for a process $F$ to be vacuum adapted is essentially equivalent to the condition $\mathbb{E} F \mathbb{E}=F$, where $\mathbb{E}$ is the conditional expectation on Fock space.

The results stated above are in their simplest form; however, we work throughout with an arbitrary (separable) initial space and in multiple Fock space of countable multiplicity.

The idea of using vacuum adaptedness in quantum stochastic calculus goes back to Hudson and Krée [9], who employed it for the investigation of processes consisting of Hilbert-Schmidt operators (which clearly cannot be adapted in the usual sense).

\subsection{Conventions}

The restriction of a function $f$ to a subset $A$ of its domain is denoted by $\left.f\right|_{A}$. The indicator function of a set $A$ is denoted by $1_{A}$ : this function equals 1 if its argument lies in the set $A$ and equals 0 otherwise. The Kronecker delta is denoted by $\delta$ : the expression $\delta_{b}^{a}$ equals 1 if $a=b$ and 0 otherwise.

All vector spaces herein have complex scalar field and all inner products are conjugate linear in their first argument. The orthogonal complement of a subset $A$ of a Hilbert space is denoted by $A^{\perp}$. The algebraic tensor product of vector spaces $X$ and $Y$ is denoted by $X \odot Y$ and $\mathrm{H} \otimes \mathrm{K}$ denotes the Hilbert-space tensor product of Hilbert spaces $\mathrm{H}$ and $\mathrm{K}$.

An operator is a densely defined, linear transformation in a Hilbert space. The closure of a set or a closable operator $A$ is denoted by $\bar{A}$. The von Neumann algebra of bounded operators on a Hilbert space $\mathrm{H}$ is denoted by $B(\mathrm{H})$ and $B(\mathrm{H} ; \mathrm{K})$ denotes the Banach space of bounded operators from a Hilbert space $\mathrm{H}$ to a Hilbert space K.

\section{Operators}

In this section we consider properties of tensor products of operators, self-adjoint operators and reduction of operators to invariant subspaces which will be used in the sequel; some routine proofs are omitted. 
Proposition 2.1. If $A, B$ and $B A$ are operators then $(B A)^{*} \supseteq A^{*} B^{*}$, with equality if $B$ is bounded.

Proof. See [19, Theorem 13.2].

\subsection{Tensor products}

If $A$ and $B$ are linear transformations in the Hilbert spaces $\mathrm{H}$ and $\mathrm{K}$, respectively, then $A \odot B$ is the linear transformation in $\mathrm{H} \otimes \mathrm{K}$ with domain $D(A) \odot D(B)$ and action on elementary tensors given by

$$
(A \odot B)(u \otimes v)=A u \otimes B v \quad \text { for all } u \in D(A) \text { and } v \in D(B) .
$$

Proposition 2.2. For any operators $A$ and $B$,

$$
A^{*} \odot B^{*} \subseteq(A \odot B)^{*} .
$$

If $A$ and $B$ are closed then $A \odot B$ is closable, with closure denoted by $A \otimes B$, and

$$
(A \otimes B)^{*}=A^{*} \otimes B^{*} .
$$

Proof. See [11, Propositions 11.2.27 and 11.2.37].

\subsection{Self-adjoint operators}

Notation. If $H$ is a self-adjoint operator in the Hilbert space $\mathrm{H}$ then

$$
E^{H}: \mathcal{B}(\mathbb{R}) \rightarrow B(\mathrm{H}) ; X \mapsto E_{X}^{H}
$$

denotes its spectral measure, where $\mathcal{B}(\mathbb{R})$ is the $\sigma$-algebra of Borel subsets of $\mathbb{R}$.

Proposition 2.3. If $A$ is an operator in the Hilbert space $\mathrm{H}$, and $U$ is an isometric isomorphism with domain $\mathrm{H}$, then

(i) $U A U^{*}$ is an operator such that $\left(U A U^{*}\right)^{*}=U A^{*} U^{*}$;

(ii) if $A$ is closable then so is $U A U^{*}$, with closure $U \bar{A} U^{*}$;

(iii) if $A$ is self-adjoint then so is $U A U^{*}$ and $U f(A) U^{*}=f\left(U A U^{*}\right)$ for any Borel-measurable function $f: \mathbb{R} \rightarrow \mathbb{C}$.

Proposition 2.4. If $H$ is a self-adjoint operator and $P$ is an orthogonal projection then $H \otimes P$ is self-adjoint and has spectral measure

$$
X \mapsto E_{X}^{K}:=E_{X}^{H} \otimes P+\delta_{0}(X) I \otimes P^{\perp},
$$

where $\delta_{0}$ denotes the Dirac measure on $\mathbb{R}$ with support $\{0\}$.

Conversely, if $H$ is a closed operator such that $H \otimes P$ is self-adjoint, where $P$ is a non-zero orthogonal projection, then $H$ is self-adjoint.

Proof. Self-adjointness of $H \otimes P$ is immediate from Proposition 2.2. It is readily verified that (2.1) defines a spectral measure; recall that $C \mapsto C \otimes D$ is strongoperator continuous on norm-bounded sets if $D$ is a bounded operator. If $X \subseteq \mathbb{R}$ is Borel, $u, v \in D(H)$ and $x, y \in D(P)$ then

$$
\left\langle u \otimes x, E_{X}^{K}(v \otimes y)\right\rangle=\left\langle u, E_{X}^{H} v\right\rangle\langle x, P y\rangle+\delta_{0}(X)\langle u, v\rangle\left\langle x, P^{\perp} y\right\rangle ;
$$


so, if $K$ denotes the self-adjoint operator with spectral measure $E^{K}$, then

$$
\begin{aligned}
\|H v\|^{2}\|P y\|^{2} & =\int_{\mathbb{R}} s^{2} d\left\langle v, E_{s}^{H} v\right\rangle\langle y, P y\rangle \\
& =\int_{\mathbb{R}} s^{2} d\left\langle v \otimes y, E_{s}^{K}(v \otimes y)\right\rangle=\int_{\mathbb{R}} s^{2} d\left\|E_{s}^{K}(v \otimes y)\right\|^{2}
\end{aligned}
$$

which shows that $v \otimes y \in D(K)$. Furthermore,

$$
\begin{aligned}
\langle u \otimes x,(H \odot P)(v \otimes y)\rangle & =\int_{\mathbb{R}} s d\left\langle u, E_{s}^{H} v\right\rangle\langle x, P y\rangle \\
& =\int_{\mathbb{R}} s d\left\langle u \otimes x, E_{s}^{K}(v \otimes y)\right\rangle=\langle u \otimes x, K(v \otimes y)\rangle,
\end{aligned}
$$

so $H \odot P \subseteq K$. Taking closures and using the fact that self-adjoint operators are maximally symmetric gives the equality claimed.

For the converse, we show first that $H$ is symmetric: let $x$ be a unit vector such that $P x=x$ and note that $D(H) \odot D(P) \subseteq D(H \otimes P)$; hence, for all $u, v \in D(H)$,

$$
\langle u, H v\rangle=\langle u \otimes x,(H \otimes P)(v \otimes x)\rangle=\langle(H \otimes P)(u \otimes x), v \otimes x\rangle=\langle H u, v\rangle .
$$

Next, suppose that $H$ is not self-adjoint; by [15, Theorem VIII.3] there exists a unit vector $w \in D\left(H^{*}\right)$ such that $H^{*} w= \pm i w$. With $x$ as before,

$$
\left(H^{*} \otimes P^{*}\right)(w \otimes x)= \pm i(w \otimes x)
$$

and therefore $H^{*} \otimes P^{*}=(H \otimes P)^{*}$ has an eigenvector with eigenvalue $\pm i$. This contradicts the fact that $H \otimes P$ is self-adjoint.

Proposition 2.5. If $H$ is a self-adjoint operator and $P$ is an orthogonal projection then $H \otimes P$ is the generator of the unitary group

$$
\mathbb{R} \ni s \mapsto U_{s}:=e^{i s H} \otimes P+I \otimes P^{\perp} .
$$

The next lemma may be proved in the same manner as [8, Theorem 10.5.4].

LEMMA 2.6. Let $U=\left(U_{s}=e^{i s H}: s \in \mathbb{R}\right)$ be a strongly continuous, oneparameter unitary group on the Hilbert space $\mathrm{H}$. If $u \in \mathrm{H}$ is such that there exists a sequence $\left(s_{n}\right)_{n=1}^{\infty} \subseteq \mathbb{R} \backslash\{0\}$ and $v \in \mathrm{H}$ with

$$
\lim _{n \rightarrow \infty} \frac{1}{i s_{n}}\left\langle w, U_{s_{n}} u-u\right\rangle=\langle w, v\rangle \quad \text { for all } w \in \mathrm{H}
$$

then $u \in D(H)$ and $H u=v$.

\subsection{Reduction}

It is fundamental to the interpretation of a non-anticipating calculus that a process can have no dependence upon the future. In mathematical terms this leads to the component operators having certain invariant subspaces.

Proposition 2.7. Let $P_{\mathrm{K}}$ denote the orthogonal projection onto $\mathrm{K}$, a closed subspace of the Hilbert space $\mathrm{H}$. If $A$ is an operator in $\mathrm{H}$ then $P_{\mathrm{K}} A \subseteq A P_{\mathrm{K}}$ if and only if

$$
P_{\mathrm{K}} D(A) \subseteq D(A), \quad A(D(A) \cap \mathrm{K}) \subseteq \mathrm{K} \quad \text { and } \quad A\left(D(A) \cap \mathrm{K}^{\perp}\right) \subseteq \mathrm{K}^{\perp} .
$$


Proof. See [1, Theorem 40.2].

Definition 2.8. If $A$ and $\mathrm{K}$ are as in Proposition 2.7 then $\mathrm{K}$ reduces $A$ and the reduction of $A$ to $\mathrm{K}$ is the operator

$$
A\left\lceil_{\mathrm{K}}: D(A) \cap \mathrm{K} \rightarrow \mathrm{K} ; u \mapsto A u .\right.
$$

(It is immediate that $A\left\lceil_{\mathrm{K}}\right.$ is densely defined: if $u \in \mathrm{K}$ then there exists a sequence $\left(u_{n}\right)_{n=1}^{\infty} \subseteq D(A)$ such that $u_{n} \rightarrow u$, but as $D\left(A \Upsilon_{\mathrm{K}}\right):=D(A) \cap \mathrm{K}=P_{\mathrm{K}} D(A)$, $\left(P_{\mathrm{K}} u_{n}\right)_{n=1}^{\infty}$ is a sequence in $D\left(A\left\lceil_{\mathrm{K}}\right)\right.$ that converges to $u$. $)$

Note that $A$ is reduced by $\mathrm{K}$ if and only if it is reduced by $\mathrm{K}^{\perp}$ and, if $B$ is also reduced by $\mathrm{K}$, so are $A+B$ and $A B$ (if $A B$ is densely defined), with $\left.(A+B)\right|_{\mathrm{K}}=$

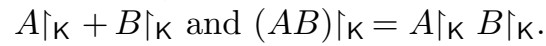

Proposition 2.9. If $A$ is a closable operator in the Hilbert space $\mathrm{H}$, and $\mathrm{K}$ is a closed subspace of $\mathrm{H}$ that reduces $A$, then $\mathrm{K}$ reduces $A^{*}$ and $\left.\left(A \uparrow_{\mathrm{K}}\right)^{*}=A^{*}\right\rceil_{\mathrm{K}}$. In particular, if $A$ is self-adjoint then $A \uparrow_{\mathrm{K}}$ is also and, if $f: \mathbb{R} \rightarrow \mathbb{C}$ is Borel measurable, $f(A)$ is reduced by $\mathrm{K}$ and $f\left(A \uparrow_{\mathrm{K}}\right)=f(A) \uparrow_{\mathrm{K}}$.

Proof. Note that

$$
P_{\mathrm{K}} A \subseteq A P_{\mathrm{K}} \Longrightarrow P_{\mathrm{K}} A^{*} \subseteq\left(A P_{\mathrm{K}}\right)^{*} \subseteq\left(P_{\mathrm{K}} A\right)^{*}=A^{*} P_{\mathrm{K}}
$$

the first inclusion and the equality follow from Proposition 2.1. If $u \in D\left(A^{*} \uparrow_{\mathrm{K}}\right)$ then

$$
\left\langle A^{*} \uparrow_{\mathrm{\kappa}} u, v\right\rangle=\left\langle A^{*} u, v\right\rangle=\langle u, A v\rangle=\left\langle u, A \uparrow_{\mathrm{k}} v\right\rangle \quad \text { for all } v \in D(A) \cap \mathrm{K},
$$

so $u \in D\left(\left(A\left\lceil_{\mathrm{K}}\right)^{*}\right)\right.$ and $\left(A\left\lceil_{\mathrm{K}}\right)^{*} u=A^{*}\right\rceil_{\mathrm{K}} u$. Conversely, if $u \in D\left(\left(A\left\lceil_{\mathrm{K}}\right)^{*}\right)\right.$ then

$$
\left\langle\left(A \uparrow_{\mathrm{K}}\right)^{*} u, v\right\rangle=\left\langle u, A \uparrow_{\mathrm{K}} v\right\rangle \quad \text { for all } v \in D\left(A \uparrow_{\mathrm{K}}\right)=D(A) \cap \mathrm{K} .
$$

If $w \in D(A)$ then $P_{\mathrm{K}} w \in D(A) \cap \mathrm{K}$ and

$$
\begin{aligned}
\langle u, A w\rangle=\left\langle u, P_{\mathrm{K}} A w\right\rangle & =\left\langle u, A P_{\mathrm{K}} w\right\rangle \\
& \left.\left.\left.=\langle u, A\rceil_{\mathrm{K}} P_{\mathrm{K}} w\right\rangle=\left\langle(A\rceil_{\mathrm{K}}\right)^{*} u, P_{\mathrm{K}} w\right\rangle=\left\langle(A\rceil_{\mathrm{K}}\right)^{*} u, w\right\rangle .
\end{aligned}
$$

Hence $u \in D\left(A^{*}\right) \cap \mathrm{K}$ and $\left.A^{*}\right|_{\mathrm{K}} u=A^{*} u=\left(A \uparrow_{\mathrm{K}}\right)^{*} u$.

That $f(A)$ is reduced by $\mathrm{K}$ if $A$ is self-adjoint follows from [22, Theorem XI.12.1] (which states that if $B$ is a bounded operator and $B A \subseteq A B$ then $B f(A) \subseteq f(A) B$ ). Finally, since $E_{X}^{A}=1_{X}(A)$ is reduced by $\left.\mathrm{K}, E_{X}^{A}\right\rceil_{\mathrm{k}}$ is well defined for all $X \in \mathcal{B}(\mathbb{R})$; it is immediate that $X \mapsto E_{X}^{A}\left\lceil\mathrm{k}\right.$ is a spectral measure and, if $u \in \mathrm{K}$ and $\left.v \in D(A\rceil_{\mathrm{K}}\right)$,

$$
\langle u, A \mid \mathrm{\kappa} v\rangle=\langle u, A v\rangle=\int_{\mathbb{R}} s d\left\langle u, E_{s}^{A} v\right\rangle=\int_{\mathbb{R}} s d\left\langle u, E_{s}^{A}\lceil\mathrm{\kappa} v\rangle .\right.
$$

Hence $A\rceil_{\mathrm{k}}$ has spectral measure $\left.X \mapsto E_{X}^{A}\right\rceil_{\mathrm{k}}$; that $f(A) \Upsilon_{\mathrm{\kappa}}=f\left(A \Upsilon_{\mathrm{\kappa}}\right)$ is immediate from this.

\subsection{Fock space}

Notation. Let $\mathcal{F}(I):=\Gamma_{+}\left(L^{2}(I ; \mathrm{k})\right)$ denote Boson (that is, symmetric) Fock space over $L^{2}(I ; \mathrm{k})$, the space of strongly measurable, square-integrable, k-valued functions on $I$, where $I$ is any Borel subset of $\mathbb{R}_{+}:=[0, \infty)$ and $\mathrm{k}$ is a separable Hilbert space, the multiplicity space. 
One way to view $\mathcal{F}(I)$ is as the closure of $\mathcal{E}(I)$, the linear span of the linearly independent family of exponential vectors $\left\{\varepsilon(u): u \in L^{2}(I ; \mathrm{k})\right\}$, with respect to the inner product

$$
\langle\varepsilon(u), \varepsilon(v)\rangle_{\mathcal{F}(I)}:=\exp \langle u, v\rangle_{L^{2}(I ; \mathrm{k})}=\exp \left(\int_{I}\langle u(t), v(t)\rangle_{\mathrm{k}} d t\right) .
$$

If $I_{1}$ and $I_{2}$ are disjoint, Borel subsets of $\mathbb{R}_{+}$then $\mathcal{F}\left(I_{1}\right) \otimes \mathcal{F}\left(I_{2}\right)$ is isometrically isomorphic to $\mathcal{F}\left(I_{1} \cup I_{2}\right)$, via $\iota_{I_{1}, I_{2}}$, the continuous extension of the linear bijection

$$
\mathcal{E}\left(I_{1}\right) \odot \mathcal{E}\left(I_{2}\right) \rightarrow \mathcal{E}\left(I_{1} \cup I_{2}\right) ; \varepsilon\left(u_{1}\right) \otimes \varepsilon\left(u_{2}\right) \mapsto \varepsilon\left(u_{1} \oplus u_{2}\right),
$$

where $u_{1} \oplus u_{2} \in L^{2}\left(I_{1} \cup I_{2} ; \mathrm{k}\right)$ is given by

$$
\left(u_{1} \oplus u_{2}\right)(t):= \begin{cases}u_{1}(t) & \text { if } t \in I_{1}, \\ u_{2}(t) & \text { if } t \in I_{2} .\end{cases}
$$

(Note that $\iota_{I_{1}, I_{2}}\left(\mathcal{E}\left(I_{1}\right) \odot \mathcal{E}\left(I_{2}\right)\right)=\mathcal{E}\left(I_{1} \cup I_{2}\right)$.)

For $t \geqslant 0$ we let $\mathcal{F}:=\mathcal{F}\left(\mathbb{R}_{+}\right), \mathcal{F}_{t}:=\mathcal{F}([0, t))$ and $\mathcal{F}^{t}:=\mathcal{F}([t, \infty))$; note that $\mathcal{F}_{t} \otimes \mathcal{F}^{t} \cong \mathcal{F}$ via the isometric isomorphism $\iota_{t}:=\iota_{[0, t),[t, \infty)}$. It is useful to introduce the notation $u_{t}:=\left.u\right|_{[0, t)}$ and $u^{t}:=\left.u\right|_{[t, \infty)}$, so that $u=u_{t} \oplus u^{t}$ and

$$
\iota_{t}^{-1} \varepsilon(u)=\varepsilon\left(u_{t}\right) \otimes \varepsilon\left(u^{t}\right) \quad \text { for all } u \in L^{2}\left(\mathbb{R}_{+} ; \mathrm{k}\right) .
$$

We also regard $u_{t}$ and $u^{t}$ as elements of $L^{2}\left(\mathbb{R}_{+} ; \mathrm{k}\right): u_{t}=1_{[0, t)} u$ and $u^{t}=1_{[t, \infty)} u$.

Definition 2.10. A subset $\mathrm{S} \subseteq L^{2}\left(\mathbb{R}_{+} ; \mathrm{k}\right)$ is admissible if

(i) $\mathcal{E}_{\mathrm{S}}:=\operatorname{lin}\{\varepsilon(u): u \in \mathrm{S}\}$ is dense in $\mathcal{F}$;

(ii) $u_{t} \in \mathrm{S}$ for all $u \in \mathrm{S}$ and $t \geqslant 0$.

We let $\mathcal{E}_{\mathrm{S}}(I):=\operatorname{lin}\left\{\varepsilon(u): u \in L^{2}(I ; \mathrm{k}) \cap \mathrm{S}\right\}$, with $L^{2}(I ; \mathrm{k})$ regarded as a subspace of $L^{2}\left(\mathbb{R}_{+} ; \mathrm{k}\right)$ in the natural manner,

$$
\mathcal{E}_{\mathrm{S}}:=\mathcal{E}_{\mathrm{S}}\left(\mathbb{R}_{+}\right), \quad \mathcal{E}_{\mathrm{S}, t}:=\mathcal{E}_{\mathrm{S}}([0, t)) \quad \text { and } \quad \mathcal{E}_{\mathrm{S}}^{t}:=\mathcal{E}_{\mathrm{S}}([t, \infty)),
$$

so that $\mathcal{E}_{\mathrm{S}}=\iota_{t}\left(\mathcal{E}_{\mathrm{S}, t} \odot \mathcal{E}_{\mathrm{S}}^{t}\right)$.

Notation. Let $\mathrm{h}$ be a separable Hilbert space (the initial space) with dense subspace $\mathrm{h}_{0}$ and define

$$
\begin{gathered}
\widetilde{\mathcal{F}}:=\mathrm{h} \otimes \mathcal{F}, \quad \widetilde{\mathcal{F}}_{t}:=\mathrm{h} \otimes \mathcal{F}_{t}, \\
\widetilde{\mathcal{E}}:=\mathrm{h}_{0} \odot \mathcal{E}\left(\mathbb{R}_{+}\right), \quad \widetilde{\mathcal{E}}_{\mathrm{S}}:=\mathrm{h}_{0} \odot \mathcal{E}_{\mathrm{S}} \quad \text { and } \quad \widetilde{\mathcal{E}}_{\mathrm{S}, t}:=\mathrm{h}_{0} \odot \mathcal{E}_{\mathrm{S}, t},
\end{gathered}
$$

so that $\widetilde{\mathcal{E}}$ and $\widetilde{\mathcal{E}}_{\mathrm{S}}$ are dense subspaces of $\widetilde{\mathcal{F}}$ and $\tilde{\iota}_{t}:=I_{\mathrm{h}} \otimes \iota_{t}$ is an isomorphism between $\widetilde{\mathcal{F}}_{t} \otimes \mathcal{F}^{t}$ and $\widetilde{\mathcal{F}}$ such that $\widetilde{\mathcal{E}}_{\mathrm{S}}=\tilde{\iota}_{t}\left(\widetilde{\mathcal{E}}_{\mathrm{S}, t} \odot \mathcal{E}_{\mathrm{S}}^{t}\right)$.

Henceforth, we omit the product sign between the components of elementary tensors in $\widetilde{\mathcal{F}}$ : if $a \in \mathrm{h}$ and $\theta \in \mathcal{F}$ then $a \theta$ denotes $a \otimes \theta \in \widetilde{\mathcal{F}}$.

Proposition 2.11. If $B$ is a closed operator in $\widetilde{\mathcal{F}}_{t}, C$ is a bounded, self-adjoint operator on $\mathcal{F}^{t}$ such that $C \varepsilon(0)=\varepsilon(0)$, and $A=\tilde{\iota}_{t}(B \otimes C) \tilde{\iota}_{t}^{*}$ then $\widetilde{\mathcal{F}}_{t}$ reduces $A$ and $A \uparrow_{\widetilde{\mathcal{F}}_{t}}=B$. 
Proof. Note that $P_{\widetilde{\mathcal{F}}_{t}}=\tilde{\iota}_{t}\left(I_{\widetilde{\mathcal{F}}_{t}} \otimes P_{\mathbb{C} \varepsilon(0)}\right) \tilde{\iota}_{t}^{*}$ : this is immediate on $\widetilde{\mathcal{E}}$, so holds everywhere by continuity. Hence

$$
P_{\widetilde{\mathcal{F}}_{t}} A=\tilde{\iota}_{t}\left(B \otimes P_{\mathbb{C} \varepsilon(0)} C\right) \tilde{\iota}_{t}^{*} \quad \text { and } \quad A P_{\widetilde{\mathcal{F}}_{t}}=\tilde{\iota}_{t}\left(B \otimes C P_{\mathbb{C} \varepsilon(0)}\right) \tilde{\iota}_{t}^{*},
$$

so $\widetilde{\mathcal{F}}_{t}$ reduces $A$ if $\mathbb{C} \varepsilon(0)$ reduces $C$, but this is trivial:

$$
P_{\mathbb{C} \varepsilon(0)} D(C)=\mathbb{C} \varepsilon(0) \subseteq \mathcal{F}^{t}=D(C), \quad C(D(C) \cap \mathbb{C} \varepsilon(0))=C \mathbb{C} \varepsilon(0)=\mathbb{C} \varepsilon(0),
$$

and if $x \in D(C) \cap \mathbb{C} \varepsilon(0)^{\perp}$ then $C x \in \mathbb{C} \varepsilon(0)^{\perp}$ because

$$
\langle C x, \gamma \varepsilon(0)\rangle=\langle x, \gamma C \varepsilon(0)\rangle=0 \text { for all } \gamma \in \mathbb{C} .
$$

For the last part, note that $\tilde{\iota}_{t}(a \varepsilon(u) \otimes \varepsilon(0))=a \varepsilon(u)$ for all $a \in \mathrm{h}$ and $u \in L^{2}[0, t)$, so $\tilde{\iota}_{t}^{*} \theta=\theta \otimes \varepsilon(0)$ for all $\theta \in \widetilde{\mathcal{E}}_{\mathrm{S}, t}$, and thus for all $\theta \in \widetilde{\mathcal{F}}_{t}$, by continuity. Hence

$$
\theta \in D(A) \cap \widetilde{\mathcal{F}}_{t} \Longleftrightarrow \theta \otimes \varepsilon(0) \in D(B \otimes C)
$$

and if $\theta \in D(B)$ then

$$
\theta \otimes \varepsilon(0) \in D(B) \odot D(C) \subseteq D(B \otimes C) ;
$$

so $\theta \in D\left(A \Gamma_{\widetilde{\mathcal{F}}_{t}}\right)$ and

$$
A \uparrow_{\widetilde{\mathcal{F}}_{t}} \theta=\tilde{\iota}_{t}(B \theta \otimes C \varepsilon(0))=B \theta .
$$

It remains to show that $D\left(A \uparrow_{\widetilde{\mathcal{F}}_{t}}\right) \subseteq D(B)$; for this, let $\theta \in D\left(A \uparrow_{\widetilde{\mathcal{F}}_{t}}\right)$ and note that $\theta \otimes \varepsilon(0) \in D(B \otimes C)$, so there exists a sequence

$$
\left(\sum_{l=1}^{m_{n}} \phi_{l}^{1, n} \otimes \phi_{l}^{2, n}\right)_{n=1}^{\infty} \subseteq D(B) \odot D(C)
$$

such that

$$
\sum_{l=1}^{m_{n}} \phi_{l}^{1, n} \otimes \phi_{l}^{2, n} \rightarrow \theta \otimes \varepsilon(0) \quad \text { and } \quad\left(\sum_{l=1}^{m_{n}} B \phi_{l}^{1, n} \otimes C \phi_{l}^{2, n}\right)_{n=1}^{\infty} \text { is convergent. }
$$

Let $\gamma_{l}^{n} \in \mathbb{C}$ be such that $P_{\mathbb{C} \varepsilon(0)} \phi_{l}^{2, n}=\gamma_{l}^{n} \varepsilon(0)$ and note that

$$
\sum_{l=1}^{m_{n}} \gamma_{l}^{n} \phi_{l}^{1, n} \otimes \varepsilon(0)=\left(I \otimes P_{\mathbb{C} \varepsilon(0)}\right)\left(\sum_{l=1}^{m_{n}} \phi_{l}^{1, n} \otimes \phi_{l}^{2, n}\right) \rightarrow \theta \otimes \varepsilon(0),
$$

from which it follows that $\sum_{l=1}^{m_{n}} \gamma_{l}^{n} \phi_{l}^{1, n} \rightarrow \theta$. Since $P_{\mathbb{C} \varepsilon(0)} C=C P_{\mathbb{C} \varepsilon(0)}$,

$$
\left(I \otimes P_{\mathbb{C} \varepsilon(0)}\right)\left(\sum_{l=1}^{m_{n}} B \phi_{l}^{1, n} \otimes C \phi_{l}^{2, n}\right)=\sum_{l=1}^{m_{n}} B \gamma_{l}^{n} \phi_{l}^{1, n} \otimes \varepsilon(0),
$$

so $\left(B \sum_{l=1}^{m_{n}} \gamma_{l}^{n} \phi_{l}^{1, n}\right)_{n=1}^{\infty}$ is convergent and thus $\theta \in D(B)$.

Proposition 2.12. If $A$ is a closable operator in $\widetilde{\mathcal{F}}$ such that $P_{\widetilde{\mathcal{F}}_{t}} A P_{\widetilde{\mathcal{F}}_{t}}=A$ then $\widetilde{\mathcal{F}}_{t}$ reduces $\bar{A}$ and

$$
\bar{A}=\tilde{\iota}_{t}\left(\bar{A} \uparrow_{\widetilde{\mathcal{F}}_{t}} \otimes P_{\mathbb{C} \varepsilon(0)}\right) \tilde{\iota}_{t}^{*}
$$

Proof. The fact that $\widetilde{\mathcal{F}}_{t}$ reduces $A$ (and so $\bar{A}$ ) is immediate from the definition. As $P_{\widetilde{\mathcal{F}}_{t}} \theta=\tilde{\iota}_{t}\left(P_{\widetilde{\mathcal{F}}_{t}} \theta \otimes \varepsilon(0)\right)$ for all $\theta \in \widetilde{\mathcal{F}}$ and $P_{\widetilde{\mathcal{F}}_{t}}=\tilde{\iota}_{t}\left(I_{\widetilde{\mathcal{F}}_{t}} \otimes P_{\mathbb{C} \varepsilon(0)}\right) \tilde{\iota}_{t}^{*}$, if $\phi \in D(A)$ then

$$
\begin{aligned}
A \phi=A \Gamma_{\widetilde{\mathcal{F}}_{t}} P_{\widetilde{\mathcal{F}}_{t}} \phi & =\tilde{\iota}_{t}\left(A \Gamma_{\widetilde{\mathcal{F}}_{t}} P_{\widetilde{\mathcal{F}}_{t}} \phi \otimes \varepsilon(0)\right) \\
& =\tilde{\iota}_{t}\left(\bar{A} \uparrow_{\widetilde{\mathcal{F}}_{t}} \otimes I_{\mathcal{F}^{t}}\right) \tilde{\iota}_{t}^{*} P_{\widetilde{\mathcal{F}}_{t}} \phi=\tilde{\iota}_{t}\left(\bar{A} \uparrow_{\widetilde{\mathcal{F}}_{t}} \otimes P_{\mathbb{C} \varepsilon(0)}\right) \tilde{\iota}_{t}^{*} \phi ;
\end{aligned}
$$


this shows that $\tilde{\iota}_{t}^{*} \bar{A} \tilde{\iota}_{t} \subseteq \bar{A} \uparrow_{\widetilde{\mathcal{F}}_{t}} \otimes P_{\mathbb{C} \varepsilon(0)}=: B$. For the reverse inclusion, let

$$
\phi_{n}=\sum_{l=1}^{m_{n}} \phi_{l}^{1, n} \otimes \phi_{l}^{2, n} \in D\left(A \Gamma_{\widetilde{\mathcal{F}}_{t}}\right) \odot D\left(P_{\mathbb{C} \varepsilon(0)}\right)=D\left(A \Gamma_{\widetilde{\mathcal{F}}_{t}}\right) \odot \mathcal{F}^{t}
$$

be such that $\phi_{n} \rightarrow \phi$ and $B \phi_{n} \rightarrow B \phi$. If $\gamma_{l}^{n} \in \mathbb{C}$ is such that $\gamma_{l}^{n} \varepsilon(0)=P_{\mathbb{C} \varepsilon(0)} \phi_{l}^{2, n}$ then $\tilde{\iota}_{t} \phi_{n} \rightarrow \tilde{\iota}_{t} \phi, P_{\widetilde{\mathcal{F}}_{t}} \tilde{\iota}_{t} \phi_{n}=\sum_{l=1}^{m_{n}} \gamma_{l}^{n} \phi_{l}^{1, n} \in D(A)$ and

$$
\tilde{\iota}_{t}^{*} A \tilde{\iota}_{t} \phi_{n}=\tilde{\iota}_{t}^{*} A P_{\widetilde{\mathcal{F}}_{t}} \tilde{\iota}_{t} \phi_{n}=\tilde{\iota}_{t}^{*} \sum_{l=1}^{m_{n}} \gamma_{n}^{l} A \phi_{l}^{1, n}=\sum_{l=1}^{m_{n}} A \Gamma_{\widetilde{\mathcal{F}}_{t}} \phi_{l}^{1, n} \otimes \gamma_{n}^{l} \varepsilon(0)=B \phi_{n},
$$

so $\tilde{\iota}_{t}^{*} A \tilde{\iota}_{t} \phi_{n} \rightarrow B \phi, \phi \in D\left(\tilde{\iota}_{t}^{*} \bar{A} \tilde{\iota}_{t}\right)$ and $\tilde{\iota}_{t}^{*} \bar{A} \tilde{\iota}_{t} \phi=B \phi$, as required.

\section{Processes}

After studying certain questions of measurability for collections of operators, we introduce the notion of process and types of adaptedness.

\subsection{Measurability}

For a function with values in a separable Hilbert space, the concepts of weak and strong measurability coincide, by a theorem of Pettis [22, Theorem V.4]. As all the Hilbert spaces occurring in this work are separable, we shall henceforth apply the adjective 'measurable' to such functions without any qualifying adverb and shall exploit both notions interchangeably.

LEMMA 3.1. If $\mathrm{H}$ is a separable Hilbert space, $\phi: \mathbb{R}_{+} \rightarrow \mathrm{H}$ is measurable and $E=(E(t): t \geqslant 0)$ is a family of operators in $\mathrm{H}$ such that

(i) $\mathbb{R}_{+} \ni t \mapsto E(t) u$ is measurable for all $u \in D(E):=\bigcap_{t \geqslant 0} D(E(t))$,

(ii) $\phi(t) \in D(E(t))$ for all $t \geqslant 0$,

(iii) $D\left(E^{*}\right)=\bigcap_{t \geqslant 0} D\left(E(t)^{*}\right)$ is dense in $\mathrm{H}$, then $\mathbb{R}_{+} \ni t \mapsto E(t) \phi(t)$ is measurable.

Proof. If $v \in D\left(E^{*}\right)$ then $\mathbb{R}_{+} \ni t \mapsto\left\langle E^{*}(t) v, u\right\rangle=\langle v, E(t) u\rangle$ is measurable for all $u \in D(E)$, so, as this set is dense, $\mathbb{R}_{+} \ni t \mapsto E^{*}(t) v$ is as well. Let $\left(\phi_{n}\right)_{n=1}^{\infty}$ and $\left(\psi_{n}\right)_{n=1}^{\infty}$ be sequences of simple functions converging almost everywhere to $\phi$ and to $\mathbb{R}_{+} \ni t \mapsto E^{*}(t) v$, respectively; $\mathbb{R}_{+} \ni t \mapsto\left\langle\psi_{n}(t), \phi_{n}(t)\right\rangle$ is a simple function for all $n$ and these converge almost everywhere to $\mathbb{R}_{+} \ni t \mapsto\left\langle E^{*}(t) v, \phi(t)\right\rangle=\langle v, E(t) \phi(t)\rangle$. Since $D\left(E^{*}\right)$ is dense in $\mathrm{H}$, the result follows.

Corollary 3.2. If $\mathrm{H}$ is a separable Hilbert space and $F, G: \mathbb{R}_{+} \rightarrow B(\mathrm{H})$ such that $\mathbb{R}_{+} \ni t \mapsto F(t) u$ and $\mathbb{R}_{+} \ni t \mapsto G(t) u$ are measurable for all $u \in \mathrm{H}$ then $\mathbb{R}_{+} \ni t \mapsto F(t) G(t) u$ is measurable for all $u \in \mathrm{H}$.

Proof. Take $E=F$ and $\phi(t)=G(t) u$ in the previous result.

If $H=(H(t): t \geqslant 0)$ is a family of self-adjoint operators, we should like the function $\mathbb{R}_{+} \ni t \mapsto f(H(t))$ to inherit some form of measurability from $H$. If $H$ consists of bounded operators then this is straightforward (and well known). 
TheOrem 3.3. Let $H=(H(t): t \geqslant 0)$ be a family of bounded, self-adjoint operators such that $\mathbb{R}_{+} \ni t \mapsto H(t) u$ is measurable for all $u \in \mathrm{H}$. If $f: \mathbb{R} \rightarrow \mathbb{C}$ is continuous then $f(H)=(f(H(t)): t \geqslant 0)$ is such that $\mathbb{R}_{+} \ni t \mapsto f(H(t)) u$ is measurable for all $u \in \mathrm{H}$.

Proof. The case where $f$ is a polynomial function is immediate, by Corollary 3.2. Next, suppose that $H$ has locally bounded norm and, for $N \in \mathbb{N}$, let

$$
\nu_{N}:=\sup \{\|H(t)\|: t \in[0, N]\} .
$$

By Weierstrass's approximation theorem, there exists a sequence of polynomials $\left(p_{n}^{N}\right)_{n=1}^{\infty}$ with $p_{n}^{N} \rightarrow f$ uniformly on $\left[-\nu_{N}, \nu_{N}\right]$ and so

$$
p_{n}^{N}(H(t)) u \rightarrow f(H(t)) u \text { for all } u \in \mathrm{H} \text { and } t \in[0, N] .
$$

Hence $\mathbb{R}_{+} \ni t \mapsto 1_{[0, N]}(t) f(H(t)) u$ is measurable for all $u \in \mathbf{H}$ and the result follows, since $1_{[0, N]} I \rightarrow I$ pointwise in norm as $N \rightarrow \infty$. Finally, if $H$ does not have locally bounded norm, let

$$
A_{n}:=\left\{t \in \mathbb{R}_{+}:\|H(t)\| \leqslant n\right\}=\|H(\cdot)\|^{-1}[0, n]
$$

and note that, because $A_{n}$ is measurable (a straightforward exercise), so too is $\mathbb{R}_{+} \ni t \mapsto 1_{A_{n}}(t) H(t) u$ for all $u \in \mathrm{H}$. As $1_{A_{n}} H$ has bounded norm, the previous working yields measurability of $\mathbb{R}_{+} \ni t \mapsto f\left(1_{A_{n}}(t) H(t)\right) u$ for all $u \in \mathbf{H}$. Finally, $f\left(1_{A_{n}}(t) H(t)\right) \rightarrow f(H(t))$ in norm as $n \rightarrow \infty$, for all $t \in \mathbb{R}_{+}$, whence the required result follows.

Corollary 3.4. If $H$ is as in Theorem 3.3 and $f: \mathbb{R} \rightarrow \mathbb{C}$ is Borel measurable then $\mathbb{R}_{+} \ni t \mapsto f(H(t)) u$ is measurable for all $u \in \bigcap_{t \geqslant 0} D(f(H(t)))$.

Proof. While this may be proved directly, it follows from the previous theorem (with $f: x \mapsto e^{i s x}$, where $s \in \mathbb{R}$ ), Theorem 3.7 and Proposition 3.6.

The technique used above is not applicable to the unbounded case, however; a stronger notion of measurability seems to be required.

LEMMA 3.5. If $H=(H(t): t \geqslant 0)$ is a collection of self-adjoint operators in the separable Hilbert space $\mathrm{H}$ and $\mathcal{M}$ denotes the class of elements $X$ in $\mathcal{B}(\mathbb{R})$ such that $\mathbb{R}_{+} \ni t \mapsto E_{X}^{H(t)} u$ is measurable for all $u \in \mathrm{H}$ then $\mathcal{M}$ is a $\sigma$-algebra.

Proof. As

$$
E_{\emptyset}^{H(t)}=0, \quad E_{\mathbb{R} \backslash X}^{H(t)}=I-E_{X}^{H(t)} \quad \text { and } \quad E_{X_{1} \cap X_{2}}^{H(t)}=E_{X_{1}}^{H(t)} E_{X_{2}}^{H(t)},
$$

we see that $\mathcal{M}$ contains $\emptyset$, is closed under complements and is closed under finite intersections, respectively; thus $\mathcal{M}$ is a subalgebra of $\mathcal{B}(\mathbb{R})$. If $\left(X_{n}\right)_{n=1}^{\infty} \subseteq \mathcal{M}$ then, without loss of generality, these sets may be assumed to be disjoint and

$$
E_{X}^{H(t)} u=\sum_{n=1}^{\infty} E_{X_{n}}^{H(t)} u \quad \text { for all } u \in \mathrm{H},
$$

so $X \in \mathcal{M}$, as required.

The next proposition indicates the correct choice for measurability. 
Proposition 3.6. Let $H=(H(t): t \geqslant 0)$ be a family of self-adjoint operators in the separable Hilbert space $\mathrm{H}$, such that $\mathbb{R}_{+} \ni t \mapsto E_{X}^{H(t)} u$ is measurable for all $u \in \mathrm{H}$ and $X \in \mathcal{B}(\mathbb{R})$. If $f: \mathbb{R} \rightarrow \mathbb{C}$ is a Borel-measurable function then $\mathbb{R}_{+} \ni t \mapsto$ $f(H(t)) u$ is measurable for all $u \in \bigcap_{t \geqslant 0} D(f(H(t)))$.

Proof. Let $f: \mathbb{R} \rightarrow \mathbb{C}$ be Borel measurable and let $\left(f_{n}\right)_{n=1}^{\infty}$ be a sequence of simple functions that converges pointwise to $f$ such that $\left|f_{n}\right| \leqslant 2|f|$ for all $n \geqslant 1$ [7, Theorem 13.5]. Since

$$
\mathbb{R}_{+} \ni t \mapsto \int_{\mathbb{R}} 1_{X}(s) d\left\langle u, E_{s}^{H(t)} v\right\rangle=\left\langle u, E_{X}^{H(t)} v\right\rangle
$$

is measurable for all $u, v \in \mathrm{H}$ and $X \in \mathcal{B}(\mathbb{R})$,

$$
\mathbb{R}_{+} \ni t \mapsto \int_{\mathbb{R}} f(s) d\left\langle u, E_{s}^{H(t)} v\right\rangle=\lim _{n \rightarrow \infty} \int_{\mathbb{R}} f_{n}(s) d\left\langle u, E_{s}^{H(t)} v\right\rangle
$$

is measurable for all $u \in \mathrm{H}$ and $v \in \bigcap_{t \geqslant 0} D(f(H(t)))$; the equality holds by the dominated-convergence theorem of Lebesgue.

ThEOREM 3.7. Let $H=(H(t): t \geqslant 0)$ be a collection of self-adjoint operators in the separable Hilbert space $\mathrm{H}$. The following are equivalent:

(i) $\mathbb{R}_{+} \ni t \mapsto(H(t)+i I)^{-1} u$ is measurable for all $u \in \mathrm{H}$;

(i') $\mathbb{R}_{+} \ni t \mapsto(H(t)+i \gamma I)^{-1} u$ is measurable for all $\gamma \in \mathbb{R} \backslash\{0\}$ and $u \in \mathrm{H}$;

(ii) $\mathbb{R}_{+} \ni t \mapsto e^{i s H(t)} u$ is measurable for all $s \in \mathbb{R}$ and $u \in \mathrm{H}$;

(iii) $\mathbb{R}_{+} \ni t \mapsto E_{X}^{H(t)} u$ is measurable for all $X \in \mathcal{B}(\mathbb{R})$ and $u \in \mathrm{H}$.

Proof. $\quad(\mathrm{i}) \Rightarrow\left(\mathrm{i}^{\prime}\right)$. Recall that if $A$ is a self-adjoint operator and $\lambda_{0} \in \mathbb{C} \backslash \mathbb{R}$, then

$$
(A+\lambda I)^{-1}=\sum_{m=0}^{\infty}\left(\lambda_{0}-\lambda\right)^{m}\left(A+\lambda_{0} I\right)^{-(m+1)} \quad \text { with }\left|\lambda-\lambda_{0}\right|<\left|\operatorname{Im} \lambda_{0}\right|
$$

(see [22, VIII.2(1),VIII.1(2)]); so the measurability of $\mathbb{R}_{+} \ni t \mapsto(H(t)+i I)^{-1}$ implies that of $\mathbb{R}_{+} \ni t \mapsto(H(t)+i \gamma I)^{-1}$ for all $\gamma \in(0,2)$ : take $\lambda_{0}=i$ and $\lambda=i \gamma$ in the above. Next, note that if $\lambda_{0}=i \gamma_{0}$ with $\gamma_{0}>0$ then the identity (3.1) holds for all $\lambda=i \gamma$ with $\gamma \in\left(0,2 \gamma_{0}\right)$; hence the result holds for all $\gamma>0$. The case where $\gamma<0$ follows by taking the adjoint.

$\left(\mathrm{i}^{\prime}\right) \Rightarrow(\mathrm{ii})$. Note that $(1-i s r / n)^{-n} \rightarrow e^{i s r}$ as $n \rightarrow \infty$ and

$$
\left|e^{i s r}-\left(1-\frac{i s r}{n}\right)^{-n}\right| \leqslant 1+\left(1+\frac{s^{2} r^{2}}{n^{2}}\right)^{-n / 2} \leqslant 2,
$$

for all $s, r \in \mathbb{R}$ and $n \geqslant 1$. Hence, by the dominated-convergence theorem,

$$
e^{i s H(t)} u=\lim _{n \rightarrow \infty}\left(I-\frac{i s H(t)}{n}\right)^{-n} u \text { for all } u \in \mathrm{H}, t \geqslant 0 .
$$

If $s \neq 0$ then

$$
\left(I-\frac{i s H(t)}{n}\right)^{-n}=\left(\frac{i n}{s}\right)^{n}\left(H(t)+i \frac{n}{s} I\right)^{-n},
$$

and so the claim follows by Corollary 3.2.

(ii) $\Rightarrow$ (iii). If $u \in \mathrm{H}$ then, since $\mathbb{R}_{+} \ni s \mapsto e^{i s H(t)} u$ is continuous for all $t \geqslant 0$, the map $\mathbb{R} \times \mathbb{R}_{+} \ni(s, t) \mapsto e^{i s H(t)} u$ is measurable, which follows from approximation 
with functions piecewise-constant in $s$ (see [7, Proof of Theorem 37.2]). If $f: \mathbb{R} \rightarrow \mathbb{C}$ is a continuous function such that it and its Fourier transform

$$
\tilde{f}: \mathbb{R} \rightarrow \mathbb{C} ; s \mapsto \frac{1}{\sqrt{2 \pi}} \int_{\mathbb{R}} f(r) e^{-i s r} d r
$$

are both Lebesgue integrable then $f$ is bounded (since $t \mapsto f(t)=\tilde{\tilde{f}}(-t)$ is continuous and tends to 0 at infinity [18, Theorem 9.11]); a simple Fubini-Tonelli argument proves that

$$
f(H(t)) u=\frac{1}{\sqrt{2 \pi}} \int_{\mathbb{R}} \tilde{f}(s) e^{i s H(t)} u d s
$$

for all $t \geqslant 0$ and that the map $\mathbb{R}_{+} \ni t \mapsto f(H(t)) u$ is measurable. Finally, for $s \in \mathbb{R}$ let $\left(f_{n}: \mathbb{R} \rightarrow[0,1]\right)_{n=1}^{\infty}$ be a sequence of infinitely differentiable functions with compact support that converges pointwise to $1_{[s, s+1]}$; such a sequence may be constructed as in $[\mathbf{1 9}, \S 1.46]$. By the dominated-convergence theorem and the previous working, we have the measurability of $\mathbb{R}_{+} \ni t \mapsto E_{[s, s+1]}^{H(t)} u$ and the result follows from Lemma 3.5.

(iii) $\Rightarrow\left(\right.$ i). This follows from Proposition 3.6 with $f(s)=(s+i)^{-1}$.

REMARK 3.8. Condition (i) of Theorem 3.7 is the measurability condition used by Reed and Simon [17, $\S$ XIII.16, p. 283]; condition (ii) is (essentially) that employed by Pathmanathan [14]. Vincent-Smith states $[\mathbf{2 0}, \S 12 ; \mathbf{2 1}, \S 2]$ that $\left(e^{i s H(t)}: t \geqslant 0\right)$ is a unitary process (as in Definition 3.14) for any $s \in \mathbb{R}$ and any self-adjoint quantum semimartingale $H$ (see $[\mathbf{2}]$ ) but provides no proof of measurability.

Definition 3.9. If $H=(H(t): t \geqslant 0)$ is a family of self-adjoint operators that satisfies the conditions of Theorem 3.7 then $H$ is spectrally measurable.

Lemma 3.10. If $H, H_{1}, H_{2}, \ldots$ are self-adjoint operators with common core D such that $H_{n} u \rightarrow H u$ for all $u \in \mathrm{D}$ then $e^{i s H_{n}} \rightarrow e^{i s H}$ strongly for all $s \in \mathbb{R}$.

Proof. These hypotheses imply the convergence of $\left(H_{n}\right)_{n=1}^{\infty}$ to $H$ in the strong resolvent sense [15, Theorem VIII.25(a)] and thus the result follows from a theorem due to Trotter [15, Theorem VIII.21].

Proposition 3.11. Let $H=(H(t): t \geqslant 0)$ be a family of self-adjoint operators in the separable Hilbert space $\mathrm{H}$ with common core $\mathrm{D}=\bigcup_{n \geqslant 1} \mathrm{D}_{n}$, where each $\mathrm{D}_{n}$ is a closed subspace of $\mathrm{H}$ and $\mathrm{D}_{n} \subseteq \mathrm{D}_{n+1}$ for all $n \geqslant 1$. If $\mathbb{R}_{+} \ni t \mapsto H(t) u$ is measurable for all $u \in \mathrm{D}$ then $H$ is spectrally measurable.

Proof. Let $P_{n}$ denote the orthogonal projection onto $\mathrm{D}_{n}$; since $\mathrm{D}$ is dense in $\mathrm{H}$, $P_{n} \rightarrow I$ strongly. If $A=H(t)$ for some $t \geqslant 0$ then $D\left(P_{n} A P_{n}\right)=D\left(A P_{n}\right)=\mathrm{H}$ and, by Proposition 2.1,

$$
\left(P_{n} A P_{n}\right)^{*} \supseteq P_{n}^{*}\left(P_{n} A\right)^{*}=P_{n} A^{*} P_{n}^{*}=P_{n} A P_{n} .
$$

Thus, by the Hellinger-Toeplitz theorem [19, Theorem 13.11(a)], $P_{n} A P_{n}$ is bounded and self-adjoint. If $u \in \mathrm{D}$ then $P_{n} u=u$ for all sufficiently large $n$; therefore $P_{n} A P_{n} u \rightarrow A u$; hence Lemma 3.10 implies that $e^{i s P_{n} A P_{n}} \rightarrow e^{i s A}$ strongly for 
all $s \in \mathbb{R}$. If $u \in \mathrm{H}$ then $P_{n} u \in \mathrm{D}_{n} \subseteq \mathrm{D}$, so $\mathbb{R}_{+} \ni t \mapsto P_{n} H(t) P_{n} u$ is measurable; thus, by Theorem 3.3, $\mathbb{R}_{+} \ni t \mapsto e^{i s \overline{P_{n}} H(t) P_{n}} u$ is also and the result follows.

EXAMPle 3.12 [4]. Recall that Boson Fock space has the chaos decomposition

$$
\mathcal{F}=\mathbb{C} \Omega \oplus \bigoplus_{n=1}^{\infty} L_{\text {sym }}^{2}\left(\mathbb{R}_{+}^{n} ; \mathrm{k}\right)=: \bigoplus_{n=0}^{\infty} \mathrm{H}_{n} .
$$

Let $p \in \mathbb{C}\langle x, y, z\rangle$ be a polynomial in the non-commuting indeterminates $x, y$ and $z$ such that $p^{\dagger}=p$, where the anti-isomorphic involution $\dagger$ satisfies $x^{\dagger}=y$ and $z=z^{\dagger}$. If $E(t)=p\left(A_{t}, A_{t}^{\dagger}, A_{t}^{\circ}\right)$ is a polynomial in the basic integrators of (one-dimensional) quantum stochastic calculus then $E(t)$ has a representation as a $*$-symmetric matrix of bounded operators, $E(t)=\left(E_{m}^{n}(t)\right)_{n, m=0}^{\infty}$, where $E_{m}^{n}(t)=E_{n}^{m}(t)^{*} \in B\left(\mathrm{H}_{m} ; \mathrm{H}_{n}\right)$ for all $m, n \in \mathbb{Z}_{+}:=\{0,1,2, \ldots\}$. Furthermore, there exists $d \in \mathbb{Z}_{+}$such that $E_{m}^{n} \equiv 0$ if $|n-m|>d$, so $D(E) \supseteq \mathrm{H}_{00}:=\sum_{n=0}^{\infty} \mathrm{H}_{n}$, the algebraic sum of the spaces $\mathrm{H}_{n}$ (the finite-particle space), and if each $E(t)$ is self-adjoint then $\mathrm{H}_{00}$ is a core for $E$. Since $t \mapsto E_{m}^{n}(t)$ is strongly continuous for all $m, n \in \mathbb{Z}_{+}$, if $E$ is self-adjoint then it is spectrally measurable.

Proposition 3.13. If $H=(H(t): t \geqslant 0)$ and $K=(K(t): t \geqslant 0)$ are each spectrally measurable collections of self-adjoint operators and $H(t)+K(t)$ is essentially self-adjoint on $D(H(t)) \cap D(K(t))$ for all $t \geqslant 0$ then the collection

$$
\overline{H+K}=(\overline{H(t)+K(t)}: t \geqslant 0)
$$

is spectrally measurable.

Proof. This follows from condition (ii) of Theorem 3.7 and Trotter's product formula [15, Theorem VIII.31].

\subsection{Processes}

Definition 3.14. If $\mathrm{H}$ is a separable Hilbert space then an $\mathrm{H}$-process $F$ is a collection of (necessarily closable) operators $(F(t): t \geqslant 0)$ in $\mathrm{H} \otimes \widetilde{\mathcal{F}}$ such that, for all $t \geqslant 0$,

(i) $D\left(1_{[0, t)} F\right)$ and $D\left(1_{[0, t)} F^{*}\right)$ are dense in $\mathrm{H} \otimes \widetilde{\mathcal{F}}$;

(ii) $\mathbb{R}_{+} \ni s \mapsto\left(1_{[0, t)} F\right)(s) \theta:=1_{[0, t)}(s) F(s) \theta$ is measurable for all $\theta \in D\left(1_{[0, t)} F\right)$. The adjoint process $F^{*}=\left(F(t)^{*}: t \geqslant 0\right)$ is also an $\mathrm{H}$-process. A process $F$ (we suppress the space $\mathrm{H}$ if it is not relevant) has core $\mathrm{D}$ if $\mathrm{D} \subseteq D(F)$ and $\overline{\left.F(t)\right|_{\mathrm{D}}}=\overline{F(t)}$ for all $t \geqslant 0$. A process $F$ is bounded or unitary if $F(t)$ is bounded or unitary, respectively, for all $t \geqslant 0$; a process $H$ is self-adjoint if $H(t)$ is self-adjoint for all $t \geqslant 0$ and $H$ is spectrally measurable.

REMARK 3.15. Note that the collection of bounded processes is a $*$-algebra and that the sum of any process and a bounded process is also a process (where all algebraic operations are defined pointwise).

REMARK 3.16. A spectrally measurable collection of self-adjoint operators is an $\mathrm{H}$-process if $D\left(1_{[0, t)} H\right)$ is dense in $\mathrm{H} \otimes \widetilde{\mathcal{F}}$ for all $t \geqslant 0$, by Proposition 3.6. 


\subsection{Adaptedness}

The continuous tensor-product structure of Fock space gives rise to notions of adaptedness: a process of operators is adapted if each term corresponds to the ampliation by some non-zero orthogonal projection. (This form is imposed by the requirement that such processes be causal, that is, non-anticipating, and for algebraic reasons.) The simplest examples involve ampliation by the identity operator (the usual, Hudson-Parthasarathy type of adaptedness) or vacuum projection (vacuum adaptedness); other possibilities have been explored (for example, Lenczewski's colour-filtered calculus [12]).

Notation. Let $p$ be an orthogonal projection onto some subspace of $\mathrm{k}$; the operator $p$ acts pointwise on $L^{2}(I ; \mathrm{k})$ in the obvious manner: $(p u)(t)=p(u(t))$ for all $t \in I$. Let $\Pi_{I}^{p}$ denote the second quantization of this operator: $\Pi_{I}^{p}$ is an orthogonal projection in $B(\mathcal{F}(I))$ and acts on $\mathcal{E}(I)$ so that $\Pi_{I}^{p} \varepsilon(u)=\varepsilon(p u)$.

Definition 3.17. An $\mathrm{H}$-process $F$ is $p$-adapted if, for all $t \geqslant 0$, there exists a closed operator $F_{t}$ in $\mathrm{H} \otimes \widetilde{\mathcal{F}}_{t}$ such that

$$
F(t) \subseteq \tilde{\iota}_{t}\left(F_{t} \otimes \Pi_{[t, \infty)}^{p}\right) \tilde{\iota}_{t}^{*}
$$

A process $F$ is strongly $p$-adapted if

$$
\bar{F}(t):=\overline{F(t)}=\tilde{\iota}_{t}\left(F_{t} \otimes \Pi_{[t, \infty)}^{p}\right) \tilde{\iota}_{t}^{*} \text { for all } t \geqslant 0 .
$$

Notation. Vacuum-adapted and HP-adapted processes are referred to as 0adapted and 1-adapted, respectively.

REMARK 3.18. If $F$ is a strongly $p$-adapted $\mathrm{H}$-process then $\bar{F}(t)$ is reduced by $\mathrm{H} \otimes \widetilde{\mathcal{F}}_{t}$ for all $t \geqslant 0$, by Proposition 2.11. The orthogonal decomposition

$$
\mathrm{H} \otimes \widetilde{\mathcal{F}}=\left(\mathrm{H} \otimes \widetilde{\mathcal{F}}_{t}\right) \oplus\left(\mathrm{H} \otimes \widetilde{\mathcal{F}}_{t}^{\perp}\right)
$$

yields a block-diagonal decomposition [1, Theorem 40.1] as follows:

$$
\bar{F}(t) \leftrightarrow\left(\begin{array}{cc}
\bar{F}(t) \Gamma_{\mathbf{H} \otimes \widetilde{\mathcal{F}}_{t}} & 0 \\
0 & \bar{F}(t)\left\lceil_{\mathbf{H} \otimes \widetilde{\mathcal{F}}_{t}^{\perp}}\right.
\end{array}\right) \quad \text { for all } t \geqslant 0 .
$$

In the particular case of 0 -adaptedness, $\left.\bar{F}(t)\right|_{\mathrm{H} \otimes \widetilde{\mathcal{F}}_{t}^{\perp}}=0$ because

$$
\bar{F}(t) P_{\mathrm{H} \otimes \tilde{\mathcal{F}}_{t}^{\perp}}=\tilde{\iota}_{t}\left(F_{t} \otimes \Pi_{[t, \infty)}^{0}\right)\left(I_{\mathrm{H} \otimes \widetilde{\mathcal{F}}_{t}} \otimes P_{\mathbb{C} \varepsilon(0)}^{\perp}\right) \tilde{\iota}_{t}^{*}=\tilde{\iota}_{t}\left(F_{t} \otimes P_{\mathbb{C} \varepsilon(0)} P_{\mathbb{C} \varepsilon(0) \perp}\right) \tilde{\iota}_{t}^{*} .
$$

In particular, if $F-I$ is strongly 0 -adapted then

$$
\bar{F}(t) \leftrightarrow\left(\begin{array}{cc}
\bar{F}(t) \Gamma_{\mathrm{H} \otimes \widetilde{\mathcal{F}}_{t}} & 0 \\
0 & I \uparrow_{\mathrm{H} \otimes \widetilde{\mathcal{F}}_{t}^{\perp}}
\end{array}\right) \quad \text { for all } t \geqslant 0 ;
$$

this explains why it is natural, in the 0 -adapted set-up, to require a unitary process $U$ to be such that $U-I$ is 0 -adapted. (Clearly $U$ cannot be 0 -adapted.)

Proposition 3.19. An $\mathrm{H}$-process $F$ such that $F=\left(I_{\mathrm{H}} \otimes \mathbb{E}\right) F\left(I_{\mathrm{H}} \otimes \mathbb{E}\right)$ is strongly 0-adapted, where the conditional expectation $\mathbb{R}_{+} \ni t \mapsto \mathbb{E}_{t} \in B(\widetilde{\mathcal{F}})$ satisfies $\mathbb{E}_{t} a \varepsilon(u)=a \varepsilon\left(u_{t}\right)$ for all $a \in \mathrm{h}$ and $u \in L^{2}\left(\mathbb{R}_{+} ; \mathrm{k}\right)$. Conversely, if $F$ is 
a strongly 0 -adapted $\mathrm{H}$-process then there is an $\mathrm{H}$-process $G$ such that

$$
G=\left(I_{\mathrm{H}} \otimes \mathbb{E}\right) G\left(I_{\mathrm{H}} \otimes \mathbb{E}\right) \quad \text { and } \quad G=\bar{F} .
$$

Proof. If $F=(I \otimes \mathbb{E}) F(I \otimes \mathbb{E})$ then, by Proposition 2.12, $F$ is strongly 0adapted. Conversely, if $F$ is strongly 0-adapted then $G: \mathbb{R}_{+} \ni t \mapsto \bar{F}(t)\left(I \otimes \mathbb{E}_{t}\right)$ is as required: Proposition 2.11 shows that $D(G(t)) \supseteq D\left(\bar{F}(t) \Gamma_{\mathbf{H} \otimes \widetilde{\mathcal{F}}_{t}}\right) \odot \mathcal{F}^{t}$ and $G(t)=\left(I_{\mathrm{H}} \otimes \mathbb{E}_{t}\right) G(t)\left(I_{\mathrm{H}} \otimes \mathbb{E}_{t}\right)$, the inclusion $D\left(G(t)^{*}\right) \supseteq D\left(F(t)^{*}\right)$ is obvious from Proposition 2.1, and measurability for $G$ holds by Lemma 3.1. Finally, $G(t)$ is closed (by Proposition 2.1) and $G(t) \Gamma_{\mathrm{H} \otimes \widetilde{\mathcal{F}}_{t}}=\bar{F}(t) \Gamma_{\mathrm{H} \otimes \widetilde{\mathcal{F}}_{t}}$.

Proposition 3.20. A p-adapted $\mathrm{H}$-process $F$ with $D(F) \supseteq \mathrm{H}_{0} \odot \widetilde{\mathcal{E}}_{\mathrm{S}}$ satisfies the inner-product identity

$$
\langle a \varepsilon(u), F(t) b \varepsilon(v)\rangle=\left\langle a \varepsilon\left(u_{t}\right), F(t) b \varepsilon\left(v_{t}\right)\right\rangle\left\langle\varepsilon\left(u^{t}\right), \varepsilon\left(p v^{t}\right)\right\rangle \quad \text { for all } t \geqslant 0,
$$

for all $a, b \in \mathrm{H}_{0} \odot \mathrm{h}_{0}$ and $u, v \in \mathrm{S}$. Conversely, an $\mathrm{H}$-process $F$ with domain or core $\mathrm{H}_{0} \odot \widetilde{\mathcal{E}}_{\mathrm{S}}$ is $p$-adapted or strongly $p$-adapted, respectively, if (3.4) holds for all $a, b \in \mathrm{H}_{0} \odot \mathrm{h}_{0}$ and $u, v \in \mathrm{S}$.

Proof. By definition, if $F$ is $p$-adapted then

$$
\langle a \varepsilon(u), F(t) b \varepsilon(v)\rangle=\left\langle a \varepsilon\left(u_{t}\right), F_{t} b \varepsilon\left(v_{t}\right)\right\rangle\left\langle\varepsilon\left(u^{t}\right), \varepsilon\left(p v^{t}\right)\right\rangle \text { for all } t \geqslant 0,
$$

for all $a, b \in \mathrm{H}_{0} \odot \mathrm{h}_{0}$ and $u, v \in \mathrm{S}$; taking $u=u_{t}$ and $v=v_{t}$ yields the identity (3.4). Conversely, (3.4) implies that $\left.F(t)\right|_{\mathrm{H}_{0} \odot \widetilde{\mathcal{E}}_{\mathrm{s}, t}}$ has range in $\mathrm{H} \otimes \widetilde{\mathcal{F}}_{t}$ (take $v=v_{t}$ ) and so

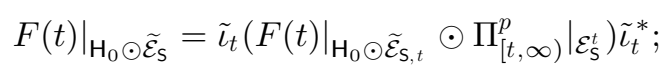

taking closures gives the result, by Propositions 2.2 and 2.3 .

Proposition 3.21. Let $H$ be a strongly p-adapted, self-adjoint $\mathrm{H}$-process. If $f: \mathbb{R} \rightarrow \mathbb{C}$ is Borel measurable with $f(0)=0$ and $f(H(t))$ has core $\mathrm{H}_{0} \odot \widetilde{\mathcal{E}}_{\mathrm{S}}$ for all $t \geqslant 0$ then $f(H)$ is a strongly $p$-adapted process.

Proof. Proposition 3.6 implies that $f(H)$ is an $\mathrm{H}$-process. Let $a, b \in \mathrm{H}_{0} \odot \mathrm{h}_{0}$ and $u, v \in \mathrm{S}$; for $t \geqslant 0$ we have

$$
\begin{aligned}
\langle a \varepsilon(u), f(H)(t) b \varepsilon(v)\rangle & =\left\langle a \varepsilon\left(u_{t}\right) \otimes \varepsilon\left(u^{t}\right), \tilde{\iota}_{t}^{*} f(H)(t) \tilde{\iota}_{t}\left(b \varepsilon\left(v_{t}\right) \otimes \varepsilon\left(v^{t}\right)\right)\right\rangle \\
& =\left\langle a \varepsilon\left(u_{t}\right) \otimes \varepsilon\left(u^{t}\right), f\left(\tilde{\iota}_{t}^{*} H(t) \tilde{\iota}_{t}\right)\left(b \varepsilon\left(v_{t}\right) \otimes \varepsilon\left(v^{t}\right)\right)\right\rangle,
\end{aligned}
$$

by Proposition 2.3. Since $H$ is strongly $p$-adapted, Propositions 2.9 and 2.11 imply that $H(t) \Upsilon_{\widetilde{\mathcal{F}}_{t}}=H_{t}$ is self-adjoint, and the last inner product above equals

$$
\begin{aligned}
\left\langle a \varepsilon\left(u_{t}\right) \otimes \varepsilon\left(u^{t}\right), f\right. & \left.\left(H_{t} \otimes \Pi_{[t, \infty)}^{p}\right)\left(b \varepsilon\left(v_{t}\right) \otimes \varepsilon\left(v^{t}\right)\right)\right\rangle \\
= & \int_{\mathbb{R}} f(s) d\left\langle a \varepsilon\left(u_{t}\right), E_{s}^{H_{t}} b \varepsilon\left(v_{t}\right)\right\rangle\left\langle\varepsilon\left(u^{t}\right), \Pi_{[t, \infty)}^{p} \varepsilon\left(v^{t}\right)\right\rangle \\
& +f(0)\left\langle a \varepsilon\left(u_{t}\right), b \varepsilon\left(v_{t}\right)\right\rangle\left\langle\varepsilon\left(u^{t}\right),\left(\Pi_{[t, \infty)}^{p}\right)^{\perp} \varepsilon\left(v^{t}\right)\right\rangle,
\end{aligned}
$$

by Proposition 2.4. As $f(0)=0$ the second term vanishes, and the first equals

$$
\left\langle a \varepsilon\left(u_{t}\right), f\left(H_{t}\right) b \varepsilon\left(v_{t}\right)\right\rangle\left\langle\varepsilon\left(u^{t}\right), \varepsilon\left(p v^{t}\right)\right\rangle=\left\langle a \varepsilon\left(u_{t}\right), f(H)(t) b \varepsilon\left(v_{t}\right)\right\rangle\left\langle\varepsilon\left(u^{t}\right), \varepsilon\left(p v^{t}\right)\right\rangle .
$$

This yields strong $p$-adaptedness, by Proposition 3.20. 
Proposition 3.22. If $H$ is a self-adjoint $\mathrm{H}$-process and $\mathbb{R}_{+} \ni t \mapsto e^{i s H(t)}-I$ is strongly $p$-adapted for all $s \in \mathbb{R}$ then $H$ is strongly p-adapted.

Proof. Since $e^{i s H}-I$ is strongly $p$-adapted, for all $t \geqslant 0$ and $s \in \mathbb{R}$ there exists a bounded operator $K_{t}(s)$ such that

$$
e^{i s H(t)}-I=\tilde{\iota}_{t}\left(K_{t}(s) \otimes \Pi_{[t, \infty)}^{p}\right) \tilde{\iota}_{t}^{*} .
$$

For the claim, it suffices to prove that, for all $t \geqslant 0$, the operator $G_{t}$, where

$$
D\left(G_{t}\right):=\left\{\theta \in \mathbf{H} \otimes \widetilde{\mathcal{F}}_{t}: \lim _{s \rightarrow 0} \frac{1}{i s} K_{t}(s) \theta \text { exists }\right\} \quad \text { and } G_{t} \theta=\lim _{s \rightarrow 0} \frac{1}{i s} K_{t}(s) \theta,
$$

is self-adjoint, since $\tilde{\iota}_{t}\left(G_{t} \odot \Pi_{[t, \infty)}^{p}\right) \tilde{\iota}_{t}^{*} \subseteq H(t)$. Equivalently, it suffices to show that $\left(K_{t}(s)+I: s \in \mathbb{R}\right)$ is a strongly continuous, one-parameter unitary group. Strong continuity is immediate from (3.5) and if

$$
M(s):=\tilde{\iota}_{t}\left(\left(K_{t}(s)+I\right) \otimes \Pi_{[t, \infty)}^{p}\right) \tilde{\iota}_{t}^{*}=e^{i s H(t)}-I+\tilde{\iota}_{t}\left(I \otimes \Pi_{[t, \infty)}^{p}\right) \tilde{\iota}_{t}^{*}
$$

then $M(s)^{*}=M(-s), M(0)=\tilde{\iota}_{t}\left(I \otimes \Pi_{[t, \infty)}^{p}\right) \tilde{\iota}_{t}^{*}$ and $M(r) M(s)=M(r+s)$ for all $r, s \in \mathbb{R}$. Since $M(s) \Gamma_{\mathrm{H} \otimes \widetilde{\mathcal{F}}_{t}}=K_{t}(s)+I$, the result follows.

Proposition 3.23. If $F$ is a strongly p-adapted $\mathrm{H}$-process then there exists a strongly 0-adapted $\mathrm{H}$-process $E$ such that $\bar{E}(t) \Gamma_{\mathrm{H} \otimes \widetilde{\mathcal{F}}_{t}}=\bar{F}(t) \uparrow_{\mathrm{H} \otimes \widetilde{\mathcal{F}}_{t}}$ for all $t \geqslant 0$, which is unique up to closure: if $G$ is a strongly 0 -adapted $\mathrm{H}$-process such that $\bar{G}(t) \uparrow_{\mathrm{H} \otimes \widetilde{\mathcal{F}}_{t}}=\bar{F}(t) \uparrow_{\mathrm{H} \otimes \widetilde{\mathcal{F}}_{t}}$ for all $t \geqslant 0$ then $\bar{G}=\bar{E}$. The process $E$ is self-adjoint if and only if $F$ has the same property.

Proof. For $t \geqslant 0$ define

$$
A(t):=\tilde{\iota}_{t}\left(\bar{F}(t) \Gamma_{\mathbf{H} \otimes \tilde{\mathcal{F}}_{t}} \odot \Pi_{[t, \infty)}^{0}\right) \tilde{\iota}_{t}^{*}=\left(I_{\mathbf{H}} \otimes \mathbb{E}_{t}\right) \tilde{\iota}_{t}\left(\bar{F}(t) \Gamma_{\mathbf{H} \otimes \widetilde{\mathcal{F}}_{t}} \odot \Pi_{[t, \infty)}^{p}\right) \tilde{\iota}_{t}^{*}
$$

and note that

$$
E(t):=\overline{A(t)} \supseteq\left(I_{\mathbf{H}} \otimes \mathbb{E}_{t}\right) \tilde{\iota}_{t}\left(\bar{F}(t) \Gamma_{\mathbf{H} \otimes \widetilde{\mathcal{F}}_{t}} \otimes \Pi_{[t, \infty)}^{p}\right) \tilde{\iota}_{t}^{*}=\left(I_{\mathbf{H}} \otimes \mathbb{E}_{t}\right) \bar{F}(t),
$$

so $D(E(t)) \supseteq D(F(t))$, and

$$
E(t)^{*}=A(t)^{*}=\left(\tilde{\iota}_{t}\left(\bar{F}(t) \Gamma_{\mathrm{H} \otimes \widetilde{\mathcal{F}}_{t}} \odot \Pi_{[t, \infty)}^{p}\right) \tilde{\iota}_{t}^{*}\left(I_{\mathrm{H}} \odot \mathbb{E}_{t}\right)\right)^{*} \supseteq\left(I_{\mathrm{H}} \otimes \mathbb{E}_{t}\right) F(t)^{*},
$$

so $D\left(E(t)^{*}\right) \supseteq D\left(F(t)^{*}\right)$. As $[0, t) \ni s \mapsto E(s) u=\left(I_{\mathrm{H}} \otimes \mathbb{E}_{s}\right) F(s) u$ is measurable if $u \in D\left(1_{[0, t)} F\right)$, by Lemma 3.1, a simple argument using the inner product and the density of $D\left(1_{[0, t)} E^{*}\right)$ yields measurability for all $u \in D\left(1_{[0, t)} E\right)$, so $E$ is an $\mathrm{H}$-process. It is also clear from the definition that

$$
E(t)=\tilde{\iota}_{t}\left(\bar{F}(t) \Gamma_{\mathbf{H} \otimes \tilde{\mathcal{F}}_{t}} \otimes \Pi_{[t, \infty)}^{0}\right) \tilde{\iota}_{t}^{*},
$$

so $E$ is strongly 0 -adapted. Uniqueness is immediate and the claim about selfadjointness is a consequence of Proposition 2.4; the necessary measurability follows from Proposition 2.5.

Definition 3.24. The closed process $E$ in Proposition 3.23 is the 0-adapted projection of the process $F$.

REMARK 3.25. If $F$ is a bounded, strongly $p$-adapted $\mathrm{H}$-process then

$$
\mathbb{R}_{+} \ni t \mapsto \tilde{\iota}_{t}\left(F(t) \Gamma_{\mathbf{H} \otimes \widetilde{\mathcal{F}}_{t}} \otimes \Pi_{[t, \infty)}^{q}\right) \tilde{\iota}_{t}^{*}
$$


is a bounded, strongly $q$-adapted process, the $q$-adapted projection of $F$, for any projection $q$; measurability may be established by the use of exponential vectors. In general, a strongly $p$-adapted process $F$ may be shown to have a strongly $q$-adapted projection, that is, (3.6) defines a process, if $q \leqslant p$ (with respect to the usual order on projections; the proof is as above) but for other $q$ it is possible that domain problems arise.

EXAMPLE 3.26. For each $t \geqslant 0$, let $H_{t}$ be a self-adjoint operator in $\mathrm{H} \otimes \widetilde{\mathcal{F}}_{t}$ with domain containing $\mathrm{H}_{0} \odot \widetilde{\mathcal{E}}_{\mathrm{S}, t}$, where $\mathrm{H}_{0}$ is dense in $\mathrm{H}$, and let

$$
H(t):=\tilde{\iota}_{t}\left(H_{t} \otimes \Pi_{[t, \infty)}^{p}\right) \tilde{\iota}_{t}^{*} .
$$

If $\mathbb{R}_{+} \ni t \mapsto\left\langle a \varepsilon\left(u_{t}\right), e^{i s H_{t}} b \varepsilon\left(v_{t}\right)\right\rangle$ is measurable for all $a, b \in \mathrm{H}_{0} \odot \mathrm{h}_{0}$ and $u, v \in \mathrm{S}$ then $H$ is a strongly $p$-adapted, self-adjoint $\mathrm{H}$-process.

\subsection{Quantum stochastic integration}

We adopt the very elegant formalism for quantum stochastic integration due to Lindsay $[\mathbf{1 3}]$. Let $\widehat{\mathrm{k}}:=\mathbb{C} \oplus \mathrm{k}, \widehat{x}:=\left(\begin{array}{l}1 \\ x\end{array}\right)$ for all $x \in \mathrm{k}$ and

$$
\widehat{f}: A \rightarrow \widehat{\mathrm{k}} ; t \mapsto \widehat{f(t)}=\left(\begin{array}{c}
1 \\
f(t)
\end{array}\right),
$$

where $f: A \rightarrow \mathrm{k}$ is any function with values in $\mathrm{k}$.

Proposition 3.27. There exists a linear contraction $\mathcal{D}: \widetilde{\mathcal{F}} \rightarrow L^{2}\left(\mathbb{R}_{+} ; \mathrm{k} \otimes \widetilde{\mathcal{F}}\right)$, the adapted gradient, such that, for all $a \in \mathrm{h}$ and $u \in L^{2}\left(\mathbb{R}_{+} ; \mathrm{k}\right)$,

$$
\mathcal{D}_{t} a \varepsilon(u):=(\mathcal{D} a \varepsilon(u))(t)=u(t) \otimes a \varepsilon\left(u_{t}\right)
$$

for almost all $t \in \mathbb{R}_{+}$.

Proof. Let $\left(e^{i}: i \in \mathbb{I}\right)$ be an orthonormal basis of $\mathrm{k}$, where $\mathbb{I}=\mathbb{N}:=\{1,2, \ldots\}$ or $\mathbb{I}=\{1, \ldots n\}$, and, for each $i \in \mathbb{I}$, define

$$
E_{i}: \widetilde{\mathcal{F}} \rightarrow \mathrm{k} \otimes \widetilde{\mathcal{F}} ; \theta \mapsto e^{i} \otimes \theta .
$$

It is readily verified that $E_{i}$ is a linear isometry and $E_{i}^{*} E_{j}=\delta_{j}^{i} I_{\widetilde{\mathcal{F}}}$ for all $i, j \in \mathbb{I}$. Suppose first that $\mathrm{h}=\mathbb{C}$ and let

$$
\mathrm{S}:=\operatorname{lin}\left\{\mathbb{R}_{+} \ni s \mapsto f(s) e^{i}: f \in L^{2}\left(\mathbb{R}_{+}\right), i \in \mathbb{I}\right\} .
$$

Define $\mathcal{D} \varepsilon(u)$, for all $u \in \mathrm{S}$, by setting

$$
\mathcal{D} \varepsilon(u): \mathbb{R}_{+} \rightarrow \mathrm{k} \otimes \mathcal{F} ;(\mathcal{D} \varepsilon(u))(t)=\mathcal{D}_{t} \varepsilon(u):=u(t) \otimes \varepsilon\left(u_{t}\right)
$$

and note that if $x \in \mathrm{k}$ and $v \in L^{2}\left(\mathbb{R}_{+} ; \mathrm{k}\right)$ then

$$
\mathbb{R}_{+} \ni t \mapsto\left\langle x \otimes \varepsilon(v), \mathcal{D}_{t} \varepsilon(u)\right\rangle=\langle x, u(t)\rangle\left\langle\varepsilon(v), \varepsilon\left(u_{t}\right)\right\rangle
$$

is measurable, so $\mathbb{R}_{+} \ni t \mapsto \mathcal{D}_{t} \varepsilon(u)$ is measurable. If $u_{i}(t):=\left\langle e^{i}, u(t)\right\rangle$ then

$$
\mathcal{D}_{t} \varepsilon(u)=\sum_{i \in I} u_{i}(t) e^{i} \otimes \varepsilon\left(u_{t}\right)=\sum_{i \in I} e^{i} \otimes u_{i}(t) \varepsilon\left(u_{t}\right)=\sum_{i \in I} E_{i}\left(\mathbb{E} D^{i}\right)_{t} \varepsilon(u),
$$

where $\mathbb{E} D^{i}$ is the composition of the conditional expectation (that is, the bounded $\mathbb{C}$-process such that $\mathbb{E}_{t} \varepsilon(u)=\varepsilon\left(u_{t}\right)$ for all $t \geqslant 0$ and $\left.u \in \mathrm{S}\right)$ and the adapted gradient 
in direction $e^{i}[\mathbf{6}, \S 5.2]$. Hence, with $\mathcal{D}$ extended by linearity, if $\theta \in \mathcal{E}_{\mathrm{S}}$ then

$$
\begin{aligned}
\left\|\mathcal{D}_{t} \theta\right\|^{2} & =\left\|\sum_{i \in I} E_{i}\left(\mathbb{E} D^{i}\right)_{t} \theta\right\|^{2} \\
& =\sum_{i, j \in I}\left\langle E_{i}\left(\mathbb{E} D^{i}\right)_{t} \theta, E_{j}\left(\mathbb{E} D^{j}\right)_{t} \theta\right\rangle=\sum_{i \in I}\left\|E_{i}\left(\mathbb{E} D^{i}\right)_{t} \theta\right\|^{2}=\sum_{i \in I}\left\|\left(\mathbb{E} D^{i}\right)_{t} \theta\right\|^{2} .
\end{aligned}
$$

By [6, Proposition 35], if $t \geqslant 0$ then

$$
\int_{0}^{t}\left\|\mathcal{D}_{s} \theta\right\|^{2} d s=\int_{0}^{t} \sum_{i \in I}\left\|\left(\mathbb{E} D^{i}\right)_{s} \theta\right\|^{2} d s=\left\|\mathbb{E}_{t} \theta\right\|^{2}-\left\|E_{0} \theta\right\|^{2} \leqslant\|\theta\|^{2},
$$

which gives the result, by the density of $\mathcal{E}_{\mathrm{S}}$. The extension to a non-trivial initial space is straightforward: if $\mathcal{D}$ is the adapted gradient acting on $\mathcal{F}=\mathbb{C} \otimes \mathcal{F}$,

$$
\tau: \mathrm{h} \otimes \mathrm{k} \otimes \mathcal{F} \rightarrow \mathrm{k} \otimes \mathrm{h} \otimes \mathcal{F}=\mathrm{k} \otimes \widetilde{\mathcal{F}} ; a \otimes b \otimes c \mapsto b \otimes a \otimes c
$$

is the isometric isomorphism given by exchanging elements of the first two spaces, and

$$
k: \mathrm{h} \otimes L^{2}\left(\mathbb{R}_{+} ; \mathrm{k} \otimes \mathcal{F}\right) \rightarrow L^{2}\left(\mathbb{R}_{+} ; \mathrm{k} \otimes \widetilde{\mathcal{F}}\right) ; k(a \otimes f)(t)=\tau(a \otimes f(t))
$$

is the natural isometric isomorphism, then $k\left(I_{\mathrm{h}} \otimes \mathcal{D}\right)=\mathcal{D}$.

Proposition 3.28. The adjoint of the adapted gradient acts isometrically on $L_{\text {ad }}^{2}\left(\mathbb{R}_{+} ; \mathrm{k} \otimes \widetilde{\mathcal{F}}\right):=\left\{f \in L^{2}\left(\mathbb{R}_{+} ; \mathrm{k} \otimes \widetilde{\mathcal{F}}\right): f(t)=\tilde{\iota}_{t}\left(f_{t} \otimes \varepsilon(0)\right)\right.$ for a.e. $\left.t \in \mathbb{R}_{+}\right\}$.

Proof. We employ the Brownian interpretation of Fock space: let $\left(e^{i}\right)_{i \in \mathbb{I}}$ be a basis for $\mathrm{k}$ and let $L^{2}(\Omega)$ be the Wiener space of a collection of independent standard Brownian motions $\left(B^{i}\right)_{i \in \mathbb{I}}$, so $\widetilde{\mathcal{F}} \cong L^{2}(\Omega ; \mathrm{h})$ via the map $k$ such that

$a \varepsilon(u) \mapsto a \mathfrak{z}(u):=a \exp \left(\sum_{i \in \mathbb{I}} \int_{\mathbb{R}_{+}} u_{i} d B^{i}-\frac{1}{2} \int_{\mathbb{R}_{+}} u_{i}^{2} d t\right)$ for all $a \in \mathrm{h}, u \in L^{2}\left(\mathbb{R}_{+} ; \mathrm{k}\right)$, where $u_{i}(t):=\left\langle e^{i}, u(t)\right\rangle$ for all $i \in \mathbb{I}$ and $t \in \mathbb{R}_{+}$. If $a, b \in \mathrm{h}, v \in L^{2}\left(\mathbb{R}_{+} ; \mathrm{k}\right)$ and $u \in L_{\text {ad }}^{2}\left(\mathbb{R}_{+} ; \mathrm{k} \otimes \mathcal{F}\right) \cong L_{\text {ad }}^{2}\left(\mathbb{R}_{+} \times \Omega ; \mathrm{k}\right)$, the $L^{2}$-space of adapted Brownian functionals, then

$$
\begin{aligned}
\langle a \otimes u, \mathcal{D}(b \varepsilon(v))\rangle & =\langle a, b\rangle \mathbb{E}\left[\int_{\mathbb{R}_{+}}\left\langle u(s), v(s) \mathfrak{z}\left(v_{s}\right)\right\rangle d s\right] \\
& =\langle a, b\rangle \mathbb{E}\left[\int_{\mathbb{R}_{+}} \sum_{i \in \mathbb{I}} \overline{u_{i}}(s) v_{i}(s) \mathfrak{z}\left(v_{s}\right) d s\right] \\
& =\langle a, b\rangle \mathbb{E}\left[\sum_{i \in \mathbb{I}} \int_{\mathbb{R}_{+}} \overline{u_{i}} d B^{i}\left(1+\sum_{j \in \mathbb{I}} \int_{\mathbb{R}_{+}} v_{j}(s) \mathfrak{z}\left(v_{s}\right) d B_{s}^{j}\right)\right] \\
& =\left\langle a \sum_{i \in \mathbb{I}} \int_{\mathbb{R}_{+}} u_{i} d B^{i}, b \mathfrak{z}(v)\right\rangle ;
\end{aligned}
$$

if desired, the issue of convergence may be finessed by choosing $u$ such that $u_{i}$ is non-zero for only finitely many $i \in \mathbb{I}$. Since

$$
\left\|\sum_{i \in \mathbb{I}} \int_{\mathbb{R}_{+}} u_{i} d B^{i}\right\|_{L^{2}(\Omega)}^{2}=\sum_{i \in \mathbb{I}} \int_{\mathbb{R}_{+}}\left|u_{i}\right|^{2} d t=\|u\|_{L^{2}\left(R_{+} ; \mathrm{k}\right)}^{2},
$$

the map $\mathcal{D}^{*}: a \otimes u \mapsto k^{-1}\left(a \sum_{i \in \mathbb{I}} \int_{\mathbb{R}_{+}} u_{i} d B^{i}\right)$ is an isometry, as claimed. 
REMARK 3.29. The two previous results may be deduced from the fact that the adapted gradient is a partial isometry with initial space $\mathrm{h} \otimes \mathbb{C} \varepsilon(0)^{\perp}$ and final space $L_{\text {ad }}^{2}\left(\mathbb{R}_{+} ; \mathrm{k} \otimes \widetilde{\mathcal{F}}\right)$, which we learned from Lindsay; the Skorohod isometry [13, Proposition 3.2] provides a short proof of this.

DEFINITION 3.30. Let

$$
\widehat{\mathcal{D}}: \widetilde{\mathcal{F}} \rightarrow L_{\text {loc }}^{2}\left(\mathbb{R}_{+} ; \widehat{\mathrm{k}} \otimes \widetilde{\mathcal{F}}\right) ;(\widehat{\mathcal{D}} \theta)(t)=\widehat{\mathcal{D}}_{t} \theta:=\left(\begin{array}{c}
\mathbb{E}_{t} \theta \\
\mathcal{D}_{t} \theta
\end{array}\right),
$$

where $L_{\text {loc }}^{2}$ refers to functions which are locally square-integrable, and note that, if $a \in \mathrm{h}$ and $u \in L^{2}\left(\mathbb{R}_{+} ; \mathrm{k}\right)$, then

$$
\widehat{\mathcal{D}}_{t} a \varepsilon(u)=\widehat{u}(t) \otimes a \varepsilon\left(u_{t}\right)
$$

for almost all $t \in \mathbb{R}_{+}$.

Notation. Let $P_{\mathrm{k}}$ denote the orthogonal projection on $\widehat{\mathrm{k}}$ with range $\mathrm{k}$ and let $\Delta:=P_{\mathrm{k}} \otimes I_{\widetilde{\mathcal{F}}}$. If $A \in B(\widehat{\mathrm{k}} \otimes \widetilde{\mathcal{F}})$, let

$$
A_{0}^{0}:=\Delta^{\perp} A \Delta^{\perp}, \quad A_{\times}^{0}:=\Delta^{\perp} A \Delta, \quad A_{0}^{\times}:=\Delta A \Delta^{\perp} \quad \text { and } \quad A_{\times}^{\times}:=\Delta A \Delta,
$$

so that

$$
A \leftrightarrow\left(\begin{array}{cc}
A_{0}^{0} & A_{\times}^{0} \\
A_{0}^{\times} & A_{\times}^{\times}
\end{array}\right)
$$

with respect to the decomposition $\widehat{k} \otimes \widetilde{\mathcal{F}}=\widetilde{\mathcal{F}} \oplus(\mathrm{k} \otimes \widetilde{\mathcal{F}})$. We extend this notation to bounded $\widehat{k}$-processes in a pointwise manner.

Notation. If $\mathbb{R}_{+} \ni t \mapsto X(t)$ is Banach-space valued and $\mathbb{R}_{+} \ni t \mapsto\|X(t)\|$ is measurable then, for all $t \geqslant 0$,

$$
\|X\|_{p, t}:= \begin{cases}\left(\int_{0}^{t}\|X(s)\|^{p} d s\right)^{1 / p} & \text { if } p \in[1, \infty), \\ \operatorname{ess} \sup \{\|X(s)\|: s \in[0, t]\} & \text { if } p=\infty\end{cases}
$$

Definition 3.31. If $F$ is a $\widehat{k}$-process then

$$
\mathcal{I}_{t}(F):=\left\{\theta \in \widetilde{\mathcal{F}}: \widehat{\mathcal{D}}_{s} \theta \in D(F(s)) \text { for almost all } s \in[0, t] \text { and }\|\theta\|_{t}^{F}<\infty\right\},
$$

where

$$
\|\theta\|_{t}^{F}:=\left\|\Delta^{\perp} F \widehat{\mathcal{D}} \theta\right\|_{1, t}+\|\Delta F \widehat{\mathcal{D}} \theta\|_{2, t},
$$

is its domain of integrability on $[0, t]$. (Measurability of $[0, t] \ni s \mapsto F(s) \widehat{\mathcal{D}}_{s} \theta$ follows from Lemma 3.1.)

THEOREM 3.32. Let $F$ be a strongly $p$-adapted $\widehat{\mathrm{k}}$-process. For all $t \geqslant 0$ there exists a linear transformation $\int F \partial \Lambda(t): \mathcal{I}_{t}(F) \rightarrow \widetilde{\mathcal{F}}$ such that

$$
\left\langle\phi, \int F \partial \Lambda(t) \theta\right\rangle=\int_{0}^{t}\left\langle\widehat{\mathcal{D}}_{s} \phi, F(s) \widehat{\mathcal{D}}_{s} \theta\right\rangle d s
$$

for all $\phi \in \widetilde{\mathcal{F}}$ and $\theta \in \mathcal{I}_{t}(F)$. If $\mathcal{I}_{t}(F)$ and $\mathcal{I}_{t}\left(F^{*}\right)$ are dense in $\widetilde{\mathcal{F}}$ for all $t \geqslant 0$ then $\int F \partial \Lambda$ is a strongly 0 -adapted $\mathbb{C}$-process, the 0 -adapted QS integral of $F$; if $F$ is 
bounded and admissible, that is,

$$
\|F\|_{t}:=\left\|F_{0}^{0}\right\|_{1, t}+\left\|F_{0}^{\times}\right\|_{2, t}+\left\|F_{\times}^{0}\right\|_{2, t}+\left\|F_{\times}^{\times}\right\|_{\infty, t}<\infty \quad \text { for all } t \geqslant 0,
$$

then $\int F \partial \Lambda$ is a strongly 0 -adapted, bounded $\mathbb{C}$-process, the equality (3.8) holds for all $\phi, \theta \in \widetilde{\mathcal{F}}$, and

$$
\left\|\int F \partial \Lambda(t)\right\| \leqslant\|F\|_{t} \quad \text { for all } t \geqslant 0
$$

Proof. If $t \geqslant 0, \theta \in \mathcal{I}_{t}(F)$ and $\phi \in \widetilde{\mathcal{F}}$ then, for almost every $s \in[0, t]$,

$$
\begin{aligned}
\left|\left\langle\widehat{\mathcal{D}}_{s} \phi, F(s) \widehat{\mathcal{D}}_{s} \theta\right\rangle\right| & \leqslant\left|\left\langle\mathbb{E}_{s} \phi, \Delta^{\perp} F(s) \widehat{\mathcal{D}}_{s} \theta\right\rangle\right|+\left|\left\langle\mathcal{D}_{s} \phi, \Delta F(s) \widehat{\mathcal{D}}_{s} \theta\right\rangle\right| \\
& \leqslant\|\phi\|\left\|\Delta^{\perp} F(s) \widehat{\mathcal{D}}_{s} \theta\right\|+\left\|\mathcal{D}_{s} \phi\right\|\left\|\Delta F(s) \widehat{\mathcal{D}}_{s} \theta\right\|=: d_{\phi, \theta}(s)
\end{aligned}
$$

and $d_{\phi, \theta}$ lies in $L^{1}[0, t]$. Thus the right-hand side of (3.8) exists and has absolute value dominated by $\|\underset{\mathcal{F}}{\|}\| \theta \|_{t}^{F}$. The Riesz-Fréchet theorem implies the existence of a unique vector $\psi \in \widetilde{\mathcal{F}}$ such that $\|\psi\| \leqslant\|\theta\|_{t}^{F}$ and

$$
\int_{0}^{t}\left\langle\widehat{\mathcal{D}}_{s} \phi, F(s) \widehat{\mathcal{D}}_{s} \theta\right\rangle d s=\langle\phi, \psi\rangle \quad \text { for all } \phi \in \widetilde{\mathcal{F}}
$$

so existence of $\int F \partial \Lambda(t) \theta$ is proven. If $\mathcal{I}_{t}(F)$ and $\mathcal{I}_{t}\left(F^{*}\right)$ are dense in $\widetilde{\mathcal{F}}$ for all $t \geqslant 0$ then we have a process: measurability of $\int F \partial \Lambda$ follows immediately from (3.8) and this equality also implies that $\left(\int F \partial \Lambda\right)^{*} \supseteq \int F^{*} \partial \Lambda$. To see that $\int F \partial \Lambda$ is strongly 0 -adapted, note that if $\theta \in \widetilde{\mathcal{F}}$ and $t \geqslant 0$ then

$$
\widehat{\mathcal{D}}_{s} \mathbb{E}_{t} \theta=\widehat{\mathcal{D}}_{s} \theta \text { for almost all } s \in[0, t],
$$

so $\mathbb{E}_{t} \theta \in \mathcal{I}_{t}(F)$ if and only if $\theta \in \mathcal{I}_{t}(F)$ and $\mathbb{E}_{t} \int F \partial \Lambda(t) \mathbb{E}_{t}=\int F \partial \Lambda(t)$. Hence $\int F \partial \Lambda(t)$ is of the required form, by Proposition 3.19. Finally, if $F$ is bounded and admissible then $\mathcal{I}_{t}(F)=\mathcal{I}_{t}\left(F^{*}\right)=\widetilde{\mathcal{F}}$ for all $t \geqslant 0$ and

$$
\begin{aligned}
\left\|d_{\phi, \theta}\right\|_{1, t} \leqslant & \|\phi\|\left\|F_{0}^{0} \mathbb{E} \theta\right\|_{1, t}+\|\phi\|\left\|F_{\times}^{0} \mathcal{D} \theta\right\|_{1, t} \\
& +\|\mathcal{D} \phi\|_{2, t}\left\|F_{0}^{\times} \mathbb{E} \theta\right\|_{2, t}+\|\mathcal{D} \phi\|_{2, t}\left\|F_{\times}^{\times} \mathcal{D} \theta\right\|_{2, t} \\
\leqslant & \|F\|_{t}\|\phi\|\|\theta\|,
\end{aligned}
$$

which gives the results claimed for this case.

REMARK 3.33. As is immediate from the previous proof, the integral $\int F \partial \Lambda$ depends only on $\left(\left.F(t)\right|_{\widehat{\mathrm{k}} \otimes \widetilde{\mathcal{F}}_{t}}: t \geqslant 0\right)$, so if $E$ is the 0 -adapted projection of $F$ then $\int E \partial \Lambda=\int F \partial \Lambda$. Vacuum adaptedness is the correct sort for the integrand, as the next remark makes clear.

REMARK 3.34. The validity of the identity

$$
\left\langle a \varepsilon(u), \int F \partial \Lambda(t) b \varepsilon(v)\right\rangle=\int_{0}^{t}\langle u(s) \otimes a \varepsilon(u), F(s) v(s) \otimes b \varepsilon(v)\rangle d s
$$

is the key property of the quantum stochastic integral (perhaps expressed in an 
unfamiliar manner); it follows from (3.8) if $F$ is 0 -adapted and has a suitable domain because

$$
\left\langle u(s) \otimes a \varepsilon\left(u_{s}\right), F(s) v(s) \otimes b \varepsilon\left(v_{s}\right)\right\rangle=\langle u(s) \otimes a \varepsilon(u), F(s) v(s) \otimes b \varepsilon(v)\rangle,
$$

and this enables us to work with the bounded adapted gradient (as opposed to the unbounded gradient operator $\nabla$ of the Malliavin calculus). The fact that 0 adaptedness is not required in the proof of Theorem 3.32 corresponds to the fact that (3.9) holds for this integral only if $F$ is 0 -adapted.

ThEOREM 3.35 (Itô product formula). If $E$ and $F$ are 0-adapted, bounded, admissible $\widehat{\mathrm{k}}$-processes, $M=\int E \partial \Lambda$, and $N=\int F \partial \Lambda$, then

$$
G:=\mathbb{R}_{+} \ni t \mapsto\left(P_{\mathrm{k}}^{\perp} \otimes M(t)\right) F(t)+E(t)\left(P_{\mathrm{k}}^{\perp} \otimes N(t)\right)+E(t) \Delta F(t)
$$

is a 0-adapted, bounded, admissible $\widehat{\mathrm{k}}$-process and $\int E \partial \Lambda \int F \partial \Lambda=\int G \partial \Lambda$.

Proof. This is a coordinate-free way of expressing a result in [6, §5], which itself follows from the usual quantum Itô product formula for bounded processes [2, Theorem 18; 13, Corollary 3.16] and the isomorphism between 0-adapted and 1-adapted quantum semimartingales $[\mathbf{6}]$.

\section{Semimartingales}

\subsection{Vacuum-adapted semimartingales}

LEMMA 4.1. If $H$ is a strongly p-adapted, self-adjoint $\mathrm{H}$-process then, for all $s \in \mathbb{R},\left(e^{i s H(t)}-I: t \geqslant 0\right)$ is a strongly $p$-adapted, bounded $\mathrm{H}$-process with uniformly bounded norm.

Proof. This follows immediately from Proposition 3.21 .

Proposition 4.2. If $A$ is a linear transformation in $\mathrm{k} \otimes \widetilde{\mathcal{F}}$ then

$$
\widehat{A}: \widetilde{\mathcal{F}} \oplus D(A) \rightarrow \widetilde{\mathcal{F}} \oplus(\mathrm{k} \otimes \widetilde{\mathcal{F}})=\widehat{\mathrm{k}} \otimes \widetilde{\mathcal{F}} ;\left(\begin{array}{c}
\theta_{0} \\
\theta_{1}
\end{array}\right) \mapsto\left(\begin{array}{c}
0 \\
A \theta_{1}
\end{array}\right)
$$

is a linear transformation in $\widehat{\mathrm{k}} \otimes \widetilde{\mathcal{F}}$ and $\widehat{A}^{*}=\widehat{A^{*}}$ if $A$ is densely defined.

Proof. We write $\theta=\left(\begin{array}{c}\theta_{0} \\ \theta_{1}\end{array}\right)$ et cetera. If $A$ is densely defined then so is $\widehat{A}$ and

$$
\left\langle\widehat{A}\left(\begin{array}{c}
\theta_{0} \\
\theta_{1}
\end{array}\right),\left(\begin{array}{c}
\psi_{0} \\
\psi_{1}
\end{array}\right)\right\rangle=\left\langle A \theta_{1}, \psi_{1}\right\rangle=\left\langle\theta_{1}, A^{*} \psi_{1}\right\rangle=\left\langle\left(\begin{array}{c}
\theta_{0} \\
\theta_{1}
\end{array}\right), \widehat{A^{*}}\left(\begin{array}{l}
\psi_{0} \\
\psi_{1}
\end{array}\right)\right\rangle
$$

for all $\theta \in D(\widehat{A})$ and $\psi \in D\left(\widehat{A^{*}}\right)$, so $\widehat{A^{*}} \subseteq \widehat{A}^{*}$. Conversely, if $\theta \in \widehat{A}^{*}$ and $\phi=\widehat{A}^{*} \theta$ then

$$
\left\langle\theta_{1}, A \psi_{1}\right\rangle=\langle\theta, \widehat{A} \psi\rangle=\langle\phi, \psi\rangle=\left\langle\phi_{0}, \psi_{0}\right\rangle+\left\langle\phi_{1}, \psi_{1}\right\rangle \quad \text { for all } \psi \in D(\widehat{A}) .
$$

Taking $\psi_{0}=0$ and $\psi_{0}=\phi_{0}$ shows that $\phi_{0}=0$, so $\theta_{1} \in D\left(A^{*}\right)$ and $A^{*} \theta_{1}=\phi_{1}$, and therefore $\theta \in D\left(\widehat{A^{*}}\right)$ with $\widehat{A^{*}} \theta=\phi=\widehat{A}^{*} \theta$, as required. 
Notation. The ${ }^{-}$notation is used to mean four different things, each of which refers to some form of extension with $\mathrm{k}$ replaced by $\widehat{k}$ :

$$
\begin{aligned}
& \widehat{x}=\left(\begin{array}{l}
1 \\
x
\end{array}\right) \in \widehat{\mathrm{k}} \text { for any vector } x \in \mathrm{k} ; \\
& \widehat{f}=t \mapsto \widehat{f(t)} \text { for any } \mathrm{k} \text {-valued function } f \\
& \widehat{\mathcal{D}} \leftrightarrow\left(\begin{array}{cc}
\mathbb{E} & 0 \\
0 & D
\end{array}\right) \text { for the adapted gradient } \mathcal{D} ; \\
& \widehat{A} \leftrightarrow\left(\begin{array}{ll}
0 & 0 \\
0 & A
\end{array}\right) \text { for any linear transformation in } \mathrm{k} \otimes \widetilde{\mathcal{F}}
\end{aligned}
$$

This should not cause confusion.

THEOREM 4.3. If $H$ is a strongly 0 -adapted, self-adjoint k-process and

$$
U_{s}:=I+\int\left(e^{i s H}-\widehat{I)} \partial \Lambda \quad \text { for } s \in \mathbb{R}\right.
$$

then, for all $t \geqslant 0$, the collection $U(t):=\left(U_{s}(t): s \in \mathbb{R}\right)$ is a strongly continuous, one-parameter unitary group.

Proof. Lemma 4.1, Theorem 3.32 and Theorem 3.35 show that $U(t)$ is a oneparameter unitary group, for all $t \geqslant 0$. To see that $\mathbb{R}_{+} \ni s \mapsto U_{s}(t)$ is weakly continuous, let $\phi, \theta \in \widetilde{\mathcal{F}}$ and note that

$$
\begin{aligned}
\left|\left\langle\phi,\left(U_{s_{1}}-U_{s_{2}}\right)(t) \theta\right\rangle\right| & \leqslant \int_{0}^{t}\left|\left\langle\mathcal{D}_{r} \phi,\left(e^{i s_{1} H(r)}-e^{i s_{2} H(r)}\right) \mathcal{D}_{r} \theta\right\rangle\right| d r \\
& \leqslant \sqrt{\int_{0}^{t}\left\|\mathcal{D}_{r} \phi\right\|^{2} d r} \sqrt{\int_{0}^{t}\left\|\left(e^{i s_{1} H(r)}-e^{i s_{2} H(r)}\right) \mathcal{D}_{r} \theta\right\|^{2} d r}
\end{aligned}
$$

as $\mathbb{R}_{+} \ni s \mapsto e^{i s H(r)}$ is strongly continuous on $\widetilde{\mathcal{F}}$ for all $r \geqslant 0$ and

$$
\left\|\left(e^{i s_{1} H(r)}-e^{i s_{2} H(r)}\right) \mathcal{D}_{r} \theta\right\|^{2} \leqslant 2\left\|\mathcal{D}_{r} \theta\right\|^{2} \in L^{1}[0, t] \quad \text { for all } s_{1}, s_{2} \in \mathbb{R},
$$

the dominated-convergence theorem gives the claim. Weak continuity implies strong continuity for unitary groups and so the result follows.

LEMMA 4.4. If $E$ is a strongly 0-adapted, bounded k-process that has locally essentially bounded norm then

$$
\int \widehat{E} \partial \Lambda(t)=\mathcal{D}^{*}\left(1_{[0, t)} E\right) \mathcal{D} \quad \text { for all } t \geqslant 0,
$$

where $1_{[0, t)} E$ acts pointwise on $L^{2}\left(\mathbb{R}_{+} ; \mathrm{k} \otimes \widetilde{\mathcal{F}}\right)$.

Proof. This follows immediately from Theorem 3.32.

THEOREM 4.5. If $H$ is a strongly 0 -adapted, self-adjoint k-process, $t \geqslant 0$ and $K(t)$ is the generator of the unitary group

$$
\mathbb{R}_{+} \ni s \mapsto U_{s}(t):=I+\int\left(e^{i s H}-\widehat{I)} \partial \Lambda(t),\right.
$$

then $K(t)=\int \widehat{H} \partial \Lambda(t)$ and $K$ is a strongly 0-adapted, self-adjoint $\mathbb{C}$-process. 
Proof. If $\theta \in D(K(t))$ then, by Lemma 4.4 and Proposition 3.28,

$$
\begin{aligned}
\lim _{s \rightarrow 0} \frac{1}{i s} \mathcal{D}^{*}\left(1_{[0, t)}\left(e^{i s H}-I\right)\right) \mathcal{D} \theta \text { exists in } \widetilde{\mathcal{F}} \\
\Longleftrightarrow \lim _{s \rightarrow 0} \frac{1}{i s}\left(e^{i s H}-I\right) \mathcal{D} \theta \text { exists in } L^{2}([0, t] ; \mathrm{k} \otimes \widetilde{\mathcal{F}}),
\end{aligned}
$$

so there exist $f \in L^{2}([0, t] ; \mathrm{k} \otimes \widetilde{\mathcal{F}})$ and a sequence $\left(s_{n}\right)_{n=1}^{\infty} \subseteq \mathbb{R} \backslash\{0\}$ such that $s_{n} \rightarrow 0$ and $\left(e^{i s_{n} H(r)}-I\right) \mathcal{D}_{r} \theta /\left(i s_{n}\right) \rightarrow f(r)$ for almost all $r \in[0, t]$. For such $r$, Lemma 2.6 implies that $\mathcal{D}_{r} \theta \in D(H(r))$ and $H(r) \mathcal{D}_{r} \theta=f(r)$, so $\theta \in \mathcal{I}_{t}(\widehat{H})$. Conversely, if $r \geqslant 0$ and $\psi \in D(H(r))$ then

$$
\lim _{s \rightarrow 0} \frac{e^{i s H(r)} \psi-\psi}{i s}=H(r) \psi
$$

and, if $s \in \mathbb{R} \backslash\{0\}$,

$$
\begin{aligned}
\left\|\frac{e^{i s H(r)} \psi-\psi}{i s}\right\|^{2} & =\int_{\mathbb{R}}\left|\frac{e^{i s t}-1}{i s}\right|^{2} d\left\|E_{t}^{H(r)} \psi\right\|^{2} \\
& =\int_{\mathbb{R}} \frac{2-2 \cos (s t)}{s^{2}} d\left\|E_{t}^{H(r)} \psi\right\|^{2} \leqslant\|H(r) \psi\|^{2} ;
\end{aligned}
$$

this inequality holds because $2(1-\cos x) \leqslant x^{2}$ for all $x \in \mathbb{R}$. Hence, if $t \geqslant 0, \phi \in \widetilde{\mathcal{F}}$ and $\theta \in \mathcal{I}_{t}(\widehat{H})$ then

$$
\begin{gathered}
\left|\left\langle\phi,\left(\frac{U_{s}(t)-I}{i s}-\int \widehat{H} \partial \Lambda(t)\right) \theta\right\rangle\right|^{2} \\
=\left|\int_{0}^{t}\left\langle\mathcal{D}_{r} \phi,\left(\frac{e^{i s H(r)}-I}{i s}-H(r)\right) \mathcal{D}_{r} \theta\right\rangle d r\right|^{2} \\
\leqslant\|\phi\|^{2} \int_{0}^{t}\left\|\left(\frac{e^{i s H(r)}-I}{i s}-H(r)\right) \mathcal{D}_{r} \theta\right\|^{2} d r
\end{gathered}
$$

by the Cauchy-Bunyakovskii-Schwarz inequality and Proposition 3.27. Thus

$$
\theta \in D(K(t)) \text { and } K(t) \theta=\int \widehat{H} \partial \Lambda(t) \theta
$$

if the final integral in (4.2) tends to zero as $s \rightarrow 0$, but this follows from the dominated-convergence theorem: the integrand converges to zero almost everywhere on $[0, t]$, by our initial working, and is bounded there by $4\left\|H(r) \mathcal{D}_{r} \theta\right\|^{2}$, using the result which follows that (and the fact that $\|x-y\|^{2} \leqslant 2\left(\|x\|^{2}+\|y\|^{2}\right.$ ) for all $x, y$ ).

Since $D(K(t))=\mathcal{I}_{t}(\widehat{H})$ is dense and $\mathcal{I}_{s}(\widehat{H}) \subseteq \mathcal{I}_{t}(\widehat{H})$ for all $s \leqslant t, D\left(1_{[0, t)} K\right)$ is dense for all $t \geqslant 0$. Spectral measurability is immediate from the definition of $K$ and strong 0-adaptedness follows from Proposition 3.22.

Definition $4.6[\mathbf{2}, \mathbf{5}]$. Let $\mathcal{S}$ and $\mathcal{S}_{\Omega}$ denote the algebras of regular quantum semimartingales on $\widetilde{\mathcal{F}}$ which are 1-adapted and 0-adapted, respectively: each $M \in$ $\mathcal{S} \cup \mathcal{S}_{\Omega}$ is a bounded process admitting the representation $M=\int F d \Lambda$ or $M=$ $\int F \partial \Lambda$, with $F$ a bounded, admissible process which is either 1- or 0-adapted. Since $M^{*}=\int F^{*} d \Lambda$ or $M^{*}=\int F^{*} \partial \Lambda$, as appropriate, $M$ is self-adjoint if and only if $F$ is self-adjoint almost everywhere. (The extension of $\mathcal{S}$ and $\mathcal{S}_{\Omega}$ to a non-trivial initial space is straightforward.) 
Corollary 4.7. If $H$ and $K$ are as in Theorem 4.5 and $K_{1}=\int H_{1} \partial \Lambda \in \mathcal{S}_{\Omega}$ is self-adjoint then $K+K_{1}$ is a strongly 0-adapted, self-adjoint process and

$$
K+K_{1}=\int\left(\widehat{H}+H_{1}\right) \partial \Lambda .
$$

Proof. This is an immediate consequence of Theorem 4.5 and the Kato-Rellich theorem [16, Theorem X.12]; measurability follows from Proposition 3.13.

COROLlary 4.8. If $H$ is a strongly 0-adapted, self-adjoint k-process then

$$
f\left(\int \widehat{H} \partial \Lambda\right)=f(0)+\int(f(H)-f(0) \widehat{)} \partial \Lambda
$$

for any bounded, Borel-measurable function $f: \mathbb{R} \rightarrow \mathbb{C}$.

Proof. If $f \in L^{1}(\mathbb{R})$ is such that $\tilde{f} \in L^{1}(\mathbb{R})$ then $f$ is bounded and

$$
\begin{aligned}
f\left(\int \widehat{H} \partial \Lambda\right)(t)-f(0) & =\frac{1}{\sqrt{2 \pi}} \int_{\mathbb{R}} \tilde{f}(s)\left(e^{i s \int \widehat{H} \partial \Lambda(t)}-I\right) d s \\
& =\frac{1}{\sqrt{2 \pi}} \int_{\mathbb{R}} \tilde{f}(s) \int\left(e^{i s H}-I\right) \widehat{)} \partial \Lambda(t) d s=: F
\end{aligned}
$$

the result follows by exchanging the order of integration: if $\phi, \theta \in \widetilde{\mathcal{F}}$ then

$$
(s, r) \mapsto\left|\tilde{f}(s)\left\langle\mathcal{D}_{r} \phi,\left(e^{i s H(r)}-I\right) \mathcal{D}_{r} \theta\right\rangle\right| \leqslant 2|\tilde{f}(s)|\left\|\mathcal{D}_{r} \phi\right\|\left\|\mathcal{D}_{r} \theta\right\| \in L^{1}(\mathbb{R} \times[0, t])
$$

and

$$
\begin{aligned}
\langle\phi, F \theta\rangle & =\frac{1}{\sqrt{2 \pi}} \int_{\mathbb{R}} \tilde{f}(s) \int_{0}^{t}\left\langle\mathcal{D}_{r} \phi,\left(e^{i s H(r)}-I\right) \mathcal{D}_{r} \theta\right\rangle d r d s \\
& =\int_{0}^{t}\left\langle\mathcal{D}_{r} \phi, \frac{1}{\sqrt{2 \pi}} \int_{\mathbb{R}} \tilde{f}(s)\left(e^{i s H(r)}-I\right) d s \mathcal{D}_{r} \theta\right\rangle d r \\
& =\int_{0}^{t}\left\langle\mathcal{D}_{r} \phi,(f(H(r))-f(0)) \mathcal{D}_{r} \theta\right\rangle d r \\
& =\left\langle\phi, \int(f(H)-f(0) \hat{)} \partial \Lambda(t) \theta\rangle .\right.
\end{aligned}
$$

If $\left(f_{n}\right)_{n=1}^{\infty}$ is a sequence of Borel-measurable functions such that $f_{n} \rightarrow f$ pointwise and $\left(\left\|f_{n}\right\|_{\infty}\right)_{n=1}^{\infty}$ is bounded then the dominated-convergence theorem implies that, for any self-adjoint operator $K$,

$$
\langle u, f(K) v\rangle=\lim _{n \rightarrow \infty} \int_{\mathbb{R}} f_{n}(x) d\left\langle u, E_{x}^{K} v\right\rangle=\lim _{n \rightarrow \infty}\left\langle u, f_{n}(K) v\right\rangle
$$

and so

$$
\left\langle\mathcal{D}_{r} \phi,\left(f_{n}(H(r))-f_{n}(0)\right) \mathcal{D}_{r} \theta\right\rangle \rightarrow\left\langle\mathcal{D}_{r} \phi,(f(H(r))-f(0)) \mathcal{D}_{r} \theta\right\rangle
$$

for almost every $r \in \mathbb{R}_{+}$. Hence the collection of bounded, Borel-measurable functions for which (4.3) holds is a unital *-algebra closed under pointwise limits of uniformly bounded sequences and containing $\left\{f \in L^{1}(\mathbb{R}): \tilde{f} \in L^{1}(\mathbb{R})\right\}$. The smallest such algebra is the set of all bounded, Borel-measurable functions $f: \mathbb{R} \rightarrow \mathbb{C}$, as required. 


\subsection{HP-adapted semimartingales}

Definition $4.9[3]$. A strongly 1 -adapted $\mathbb{C}$-process $M$ of closable operators is the 1-adapted $Q S$ integral of the strongly 1-adapted $\widehat{k}$-process $F$, denoted by $M=\int F d \Lambda$, if, for all $t \geqslant 0, \widetilde{\mathcal{F}}_{t}$ reduces $M(t)$ and

$$
M(t) \Upsilon_{\widetilde{\mathcal{F}}_{t}}=\int\left(F+P_{\mathrm{k}} \otimes \bar{M}\right) \partial \Lambda(t) \uparrow_{\widetilde{\mathcal{F}}_{t}}
$$

where $\int\left(F+P_{\mathrm{k}} \otimes \bar{M}\right) \partial \Lambda$ is the 0 -adapted integral of Theorem 3.32. If $\widetilde{\mathcal{E}}_{\mathrm{S}} \subseteq D(M)$ then

$$
\begin{aligned}
\left\langle a \varepsilon\left(u_{t}\right), M(t) b \varepsilon\left(v_{t}\right)\right\rangle= & \int_{0}^{t}\left\langle\widehat{\mathcal{D}}_{s} \varepsilon\left(u_{t}\right),\left(F+P_{\mathrm{k}} \otimes \bar{M}\right)(s) \widehat{\mathcal{D}}_{s} \varepsilon\left(v_{t}\right)\right\rangle d s \\
= & \int_{0}^{t}\left\langle\widehat{u}(s) \otimes a \varepsilon\left(u_{s}\right), F(s) \widehat{v}(s) \otimes b \varepsilon\left(v_{s}\right)\right\rangle d s \\
& +\int_{0}^{t}\langle u(s), v(s)\rangle\left\langle a \varepsilon\left(u_{s}\right), M(s) b \varepsilon\left(v_{s}\right)\right\rangle d s
\end{aligned}
$$

and it is a simple exercise to verify that

$$
\langle a \varepsilon(u), M(t) b \varepsilon(v)\rangle=\int_{0}^{t}\langle\widehat{u}(s) \otimes a \varepsilon(u), F(s) \widehat{v}(s) \otimes b \varepsilon(v)\rangle d s .
$$

In particular, this definition agrees with the usual one in the case of regular quantum semimartingales $[\mathbf{2}]$.

REMARK 4.10. If $E$ is strongly 1-adapted and $M=\int E d \Lambda$ is its 1-adapted integral then, if $N$ and $F$ are the 0-adapted projections of $M$ and $E$, respectively, $N=\int\left(F+P_{\mathrm{k}} \otimes N\right) \partial \Lambda$. (More in this vein may be found in [6].)

Proposition 4.11. If $H$ is a strongly 1-adapted, self-adjoint k-process then let $G$ be the 0-adapted projection of $H$ and let

$$
U_{s}:=I+\int\left(e^{i s G}-\widehat{I)} \partial \Lambda \quad \text { for all } s \in \mathbb{R} .\right.
$$

If $V_{s}-I$ is the 1-adapted projection of $U_{s}-I$ then $V_{s}$ is a strongly 1-adapted, unitary $\mathbb{C}$-process and

$$
V_{s}=I+\int\left(e^{i s H}-I \otimes V_{s} \widehat{)} d \Lambda \quad \text { for all } s \in \mathbb{R}\right.
$$

Proof. The process $U_{s}$ is unitary, for all $s \in \mathbb{R}$, by Theorem 4.3, and therefore $V_{s}(t)$ is unitary for all $(s, t) \in \mathbb{R} \times \mathbb{R}_{+}$:

$$
V_{s}(t)=I+\tilde{\iota}_{t}\left(\left(U_{s}(t)-I\right) \uparrow_{\tilde{\mathcal{F}}_{t}} \otimes \Pi_{[t, \infty)}^{1}\right) \tilde{\iota}_{t}^{*}=\tilde{\iota}_{t}\left(U_{s}(t) \uparrow_{\tilde{\mathcal{F}}_{t}} \otimes \Pi_{[t, \infty)}^{1}\right) \tilde{\iota}_{t}^{*},
$$

so each $V_{s}$ is a strongly 1 -adapted, unitary $\mathbb{C}$-process, as claimed. Furthermore, by [6, Corollary 40],

$$
\left.\left(V_{s}-I\right)\right|_{\widetilde{\mathcal{E}}_{\mathrm{S}}}=\int\left(\left(e^{i s H}-I\right)-\left.I \otimes\left(V_{s}-I\right) \widehat{)} d \Lambda\right|_{\widetilde{\mathcal{E}}_{\mathrm{S}}}\right.
$$

for the admissible subset $\mathrm{S}=\operatorname{lin}\left\{f e^{i}: f \in L^{2}\left(\mathbb{R}_{+}\right), i \in \mathbb{I}\right\}$, where $\left(e^{i}: i \in \mathbb{I}\right)$ is an orthonormal basis for $\mathrm{k}$; this holds because the 1-adapted projection of $e^{i s G}-I$ is $e^{i s H}-I$, by Proposition 2.5. As $\mathcal{I}_{t}\left(e^{i s H}-I \otimes V_{s}\right)=\widetilde{\mathcal{F}}$ for all $t \geqslant 0$, the result follows. 
Theorem 4.12. If $H$ and $V$ are as in Proposition 4.11 and, for all $t \geqslant 0$,

$$
K(t):=\tilde{\iota}_{t}\left(\int \widehat{H} \partial \Lambda(t) \Gamma_{\widetilde{\mathcal{F}}_{t}} \otimes \Pi_{[t, \infty)}^{1}\right) \tilde{\iota}_{t}^{*}
$$

then

$$
K(t)=\lim _{s \rightarrow 0} \frac{V_{s}(t)-I}{i s},
$$

where the limit is taken in the strong sense, and $K$ is a strongly 1-adapted, selfadjoint $\mathbb{C}$-process such that, if $D\left(1_{[0, t)}(H-I \otimes K)\right)$ is dense in $\mathrm{k} \otimes \widetilde{\mathcal{F}}$ for all $t \geqslant 0$,

$$
K=\int(H-I \otimes K \widehat{)} d \Lambda .
$$

Proof. If $U$ and $G$ are as in Proposition 4.11 then

$$
\int \widehat{G} \partial \Lambda(t)=\tilde{\iota}_{t}\left(\int \widehat{G} \partial \Lambda(t) \Gamma_{\widetilde{\mathcal{F}}_{t}} \otimes \Pi_{[t, \infty)}^{0}\right) \tilde{\iota}_{t}^{*},
$$

so $\left.\int \widehat{G} \partial \Lambda(t)\right|_{\widetilde{\mathcal{F}}_{t}}$ is self-adjoint and, since $\widehat{G}$ is the 0 -adapted projection of $\widehat{H}$,

$$
K(t)=\tilde{\iota}_{t}\left(\int \widehat{G} \partial \Lambda(t)\left\lceil_{\tilde{\mathcal{F}}_{t}} \otimes \Pi_{[t, \infty)}^{1}\right) \tilde{\iota}_{t}^{*} .\right.
$$

By Proposition 2.5, this is the generator of

$$
\mathbb{R} \ni s \mapsto \tilde{\iota}_{t}\left(U_{s}(t) \Gamma_{\widetilde{\mathcal{F}}_{t}} \otimes \Pi_{[t, \infty)}^{1}\right) \tilde{\iota}_{t}^{*}=V_{s}(t),
$$

and the final result follows if $H-I \otimes K$ is a k-process, which is an immediate consequence of the domain condition.

EXAMPLE 4.13. If $H$ is a strongly 1-adapted, self-adjoint k-process such that $\mathrm{k}_{0} \odot \widetilde{\mathcal{E}}_{\mathrm{S}} \subseteq D(H(t))$ for all $t \geqslant 0$, where $\mathrm{k}_{0}$ is a dense subspace of $\mathrm{k}$ and

$$
\mathbb{R}_{+} \ni t \mapsto H(t)\left(u(t) \otimes \varepsilon\left(u_{t}\right)\right) \in L_{\text {loc }}^{2}\left(\mathbb{R}_{+} ; \mathrm{k}\right) \quad \text { for all } u \in \mathrm{S},
$$

then $\mathrm{k}_{0} \odot \widetilde{\mathcal{E}}_{\mathrm{S}} \subseteq D(H(t)-I \otimes K(t))$ for all $t \geqslant 0$, since

$$
D(K(t)) \supseteq D\left(\int \widehat{H} \partial \Lambda(t)\left\lceil_{\widetilde{\mathcal{F}}_{t}}\right) \odot \mathcal{F}^{t} \supseteq\left(\mathcal{I}_{t}(\widehat{H}) \cap \widetilde{\mathcal{F}}_{t}\right) \odot \mathcal{E}_{\mathrm{S}}^{t} \supseteq \widetilde{\mathcal{E}}_{\mathrm{S}, t} \odot \mathcal{E}_{\mathrm{S}}^{t}=\widetilde{\mathcal{E}}_{\mathrm{S}}\right.
$$

Corollary 4.9. If $H$ and $K$ are as in Theorem 4.12, with $D\left(1_{[0, t)}(H-I \otimes K)\right)$ dense in $\mathrm{k} \otimes \widetilde{\mathcal{F}}$ for all $t \geqslant 0$, and $K_{1}=\int H_{1} d \Lambda \in \mathcal{S}$ is self-adjoint then $K+K_{1}$ is a strongly 1-adapted, self-adjoint $\mathbb{C}$-process and

$$
K+K_{1}=\int\left(\left(H-I \otimes K \widehat{)}+H_{1}\right) d \Lambda\right.
$$

Proof. This is the same as for Corollary 4.7.

Acknowledgements. The initial stages of this work were carried out when the author was at Lady Margaret Hall, University of Oxford, and it was completed while he was visiting Strasbourg with financial support provided through the European Community's Human Potential Programme under contract HPRN-CT-2002-00279, QP-Applications; the support of all these organisations is gratefully acknowledged. Thanks are also extended to Dr J. M. Lindsay, who provided some useful comments on a previous draft and caused a serious misapprehension to be rectified, and to the referee. 


\section{References}

1. N. I. Akhiezer and I. M. Glazman, Theory of linear operators in Hilbert space I (Frederick Ungar, New York, 1961).

2. S. Attal, 'An algebra of non-commutative bounded semimartingales: square and angle quantum brackets', J. Funct. Anal. 124 (1994) 292-332.

3. S. Attal, 'Extensions of quantum stochastic calculus', Quantum probability communications XI, Grenoble summer school, 1998 (ed. S. Attal and J. M. Lindsay; World Scientific, Singapore, 2003) 1-37.

4. A. C. R. Belton, 'A matrix formulation of quantum stochastic calculus', DPhil Thesis, University of Oxford, 1998.

5. A. C. R. Belton, 'Quantum $\Omega$-semimartingales and stochastic evolutions', J. Funct. Anal. 187 (2001) 94-109.

6. A. C. R. Belton, 'An isomorphism of quantum semimartingale algebras', Q. J. Math. 55 (2004) 135-165.

7. P. Billingsley, Probability and measure, 3rd edn (John Wiley, New York, 1995).

8. E. Hille and R. S. Phillips, Functional analysis and semi-groups, revised edn, Colloquium Publications 31 (American Mathematical Society, Providence, RI, 1957).

9. R. L. Hudson and P. KRÉE, 'Quantum stochastic calculus for Hilbert Schmidt processes', Stochastic analysis, path integration and dynamics, Warwick, 1987 (ed. K. D. Elworthy and J.-C. Zambrini), Pitman Research Notes in Mathematics 200 (Longman Scientific \& Technical, Harlow, 1989) 83-93.

10. R. L. Hudson and K. R. Parthasarathy, 'Quantum Ito's formula and stochastic evolutions', Comm. Math. Phys. 93 (1984) 301-323.

11. R. V. KAdison and J. R. Ringrose, Fundamentals of the theory of operator algebras II: advanced theory, Graduate Studies in Mathematics 16 (American Mathematical Society, Providence, RI, 1997).

12. R. Lenczewski, 'Filtered stochastic calculus', Infin. Dimens. Anal. Quantum Probab. Relat. Top. 4 (2001) 309-346.

13. J. M. Lindsay, 'Quantum stochastic analysis - an introduction', Quantum independent increment processes I (eds M. Schürmann and U. Franz), Lecture Notes in Mathematics 1865 (Springer, Berlin, 2005) 181-271.

14. S. Pathmanathan, 'The Poisson process in quantum stochastic calculus', DPhil Thesis, University of Oxford, 2002.

15. M. REeD and B. Simon, Methods of modern mathematical physics I: functional analysis (Academic Press, New York, 1972).

16. M. REED and B. Simon, Methods of modern mathematical physics II: Fourier analysis, selfadjointness (Academic Press, New York, 1975).

17. M. ReED and B. Simon, Methods of modern mathematical physics IV: analysis of operators (Academic Press, New York, 1978).

18. W. Rudin, Real and complex analysis, 3rd edn (McGraw-Hill, New York, 1987).

19. W. Rudin, Functional analysis, 2nd edn (McGraw-Hill, New York, 1991).

20. G. F. Vincent-Smith, 'The Itô formula for quantum semimartingales', Proc. London Math. Soc. (3) 75 (1997) 671-720.

21. G. F. Vincent-Smith, 'The Itô formula for perturbed Brownian martingales', Bull. London Math. Soc. 32 (2000) 736-745.

22. K. YosidA, Functional analysis, 6th edn (Springer, Berlin, 1980).

\author{
Alexander C. R. Belton \\ IRMA \\ 7 rue René Descartes \\ 67084 Strasbourg cedex \\ France
}

\author{
Current address: \\ School of Mathematics, Applied \\ Mathematics and Statistics \\ University College \\ Cork \\ Ireland
}

a.belton@ucc.ie 